CMU-HEP 97-07

UCSD/PTH 97-12

\title{
Precision Corrections to Dispersive Bounds on Form Factors
}

\author{
C. Glenn Boyd* \\ Department of Physics, Carnegie Mellon University \\ Benjamín Grinstein ${ }^{\dagger}$ \\ and \\ Richard F. Lebed ${ }^{\ddagger}$ \\ Department of Physics, University of California at San Diego, La Jolla, CA 92093
}

(May, 1997)

\begin{abstract}
We present precision corrections to dispersion relation bounds on form factors in bottom hadron semileptonic decays and analyze their effects on parameterizations derived from these bounds. We incorporate QCD two-loop and nonperturbative corrections to the two-point correlator, consider form factors whose contribution to decay rates is suppressed by lepton mass, and implement more realistic estimates of truncation errors associated with the parameterizations. We include higher resonances in the hadronic sum that,
\end{abstract}

\footnotetext{
*boyd@fermi.phys.cmu.edu

†bgrinstein@ucsd.edu

${ }_{\ddagger}^{\ddagger}$ rlebed@ucsd.edu
} 
together with heavy quark symmetry relations near zero recoil, further tighten the sum rule bounds. Utilizing all these improvements, we show that each of the six form factors in $\bar{B} \rightarrow D \ell \bar{\nu}$ and $\bar{B} \rightarrow D^{*} \ell \bar{\nu}$ can be described with $3 \%$ or smaller precision using only the overall normalization and one unknown parameter. A similar one-coefficient parameterization of one of the $\Lambda_{b} \rightarrow \Lambda_{c} \ell \bar{\nu}$ form factors, together with heavy quark symmetry relations valid to order $1 / m^{2}$, describes the differential baryon decay rate in terms of one unknown parameter and the phenomenologically interesting quantity $\bar{\Lambda}_{\Lambda} \approx M_{\Lambda_{b}}-m_{b}$. We discuss the validity of slope-curvature relations derived by Caprini and Neubert, and present weaker, corrected relations. Finally, we present sample fits of current experimental $\bar{B} \rightarrow D^{*} \ell \bar{\nu}$ and $\bar{B} \rightarrow D \ell \bar{\nu}$ data to the improved one-parameter expansion.

Typeset using REVTEX 


\section{INTRODUCTION}

The decays of bottom hadrons provide fertile ground for explorations of both weak and strong interactions. The empirical smallness of the CKM elements $\left|V_{c b}\right|$ and $\left|V_{u b}\right|$ implies that $b$-hadron decays are relatively long-lived, and thus more easily analyzed, while the heaviness of the $b$ quark means that the heavy quark effective theory (HQET) [1, 2] provides a reliable expansion for describing the approximate decoupling of the $b$ quark from the rest of the hadron, leading to a substantial simplification of the strong-interaction dynamics.

Semileptonic decays of $b$-hadrons are particularly tractable from the theoretical point of view, since the leptonic current may be trivially separated from the hadronic current. Furthermore, focusing upon exclusive decays permits one to avoid questions regarding the validity of quark-hadron duality near kinematic endpoint regions. On the other hand, exclusive processes must be described in terms of a number of nonperturbative form factors that encapsulate the physics of the hadronization process.

HQET has provided a substantial leap forward in the exclusive analysis, demonstrating that heavy-to-heavy quark transitions at zero recoil are accompanied by a complete overlap of initial- and final-state hadron wave functions [1, 3]. Consequently, each form factor possesses a well-defined normalization at this kinematic point. For the case of $\bar{B}^{(*)} \rightarrow D^{(*)}$ transitions, for example, each of the 20 form factors either vanishes or is proportional to a single universal form factor $\xi$, which represents this wave function overlap as a function of momentum

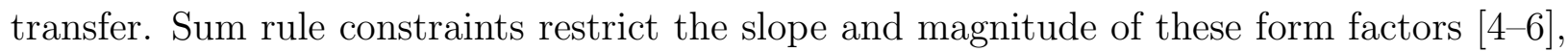
but otherwise shapes of the form factors are à priori undetermined functions of momentum transfer.

However, some recent work [0] 18] has demonstrated that one may obtain rather nontrivial and model-independent constraints on the shape of such form factors in the context of dispersion relations. Using basic field theoretic properties like causality and crossing symmetry, one finds that the shape of a given form factor is determined to high accuracy over its entire kinematic range by its value at only a small number of points $\square$ 10. Indeed, for 
the case of $\bar{B} \rightarrow D^{(*)} \ell \bar{\nu}$, the HQET normalization plus only one additional point determines most of the form factors to 3-7\% accuracy [7]. Similar analyses provide interesting and useful constraints on $\bar{B} \rightarrow \pi \ell \bar{\nu}$ [7, 10 [13], $\bar{B} \rightarrow \rho \ell \bar{\nu}$ [14], and $\Lambda_{b} \rightarrow \Lambda_{c} \ell \bar{\nu}$ [7].

At the core of this analysis lies a dispersion relation in momentum space that relates the integral of an inclusive production rate to a two-point function evaluated far from physical resonances, where perturbative QCD is quantitatively reliable. The production rate is a sum of positive-definite exclusive rates; it follows that all contributions to the hadronic side serve to saturate more and more closely the bound from the perturbative side. Clearly, two improvements to this procedure include a better calculation of the QCD side (which includes both multi-loop effects and nonperturbative vacuum condensates), and the inclusion of more states on the hadronic side. The former may be extracted from existing literature, while the latter may be achieved using heavy quark symmetry relations. The inclusion of higher states has previously been investigated for elastic $B \rightarrow B$ scattering [17] and for $\bar{B} \rightarrow \pi \ell \bar{\nu}$ decays [11], but not for $b \rightarrow c$ transitions. For mesonic and baryonic $b \rightarrow c$ transitions, the inclusion of higher states improves considerably the precision of the dispersion-relation constraints, and represents the primary contribution of this work. In addition, we include in this analysis nonzero lepton masses, anticipating future measurements of processes such as $\bar{B} \rightarrow D^{(*)} \tau \bar{\nu}_{\tau}$ or $\bar{B} \rightarrow \pi \tau \bar{\nu}_{\tau}$.

This paper is organized as follows. In Sec. II we define the form factors for the physicallyobserved semileptonic decays and present expressions for differential decay widths including lepton masses. Section III presents a brief review of the dispersive method for obtaining bounds on the form factors. Ingredients of the form factor parameterizations, including explicit formulas for the QCD results at one and two loops as well as leading nonperturbative effects, appear in Sec. IV, followed by a tabulation of the form factor weighting functions, which are central to the dispersive method. In Sec. $\mathrm{V}$ we discuss the inclusion of additional, previously ignored hadronic states into the dispersion relations, and show how heavy quark effective theory may be used to include their effects and thereby tighten form factor con- 


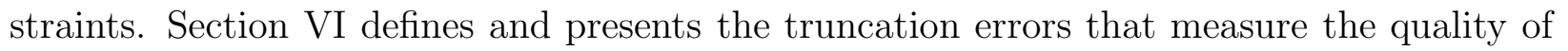
our form factor parameterizations. In Sec. $\nabla I I$ we discuss slope-curvature relations derived by Caprini and Neubert [16], point out an invalidating assumption, and examine the form of the corrected relations. In Sec. VIII we present the results of the current analysis in fits to the latest experimental data, and in Sec. $[\mathrm{X}]$ we conclude.

\section{FORM FACTORS}

We begin by defining form factors for the semileptonic decays of interest in terms of hadronic transition matrix elements. From the field theory point of view, it is most convenient to define form factors as coefficients of independent Lorentz structures appearing in the matrix element. However, the combinations of form factors most easily obtained from data are those appearing in a sum of squares in the differential rates, namely, the helicity amplitudes. As seen below, the helicity amplitudes are particular linear combinations of the original form factors, and thus simply form a different basis for the description of the matrix elements. It is these helicity amplitudes we wish to constrain.

The notation used throughout the paper is as follows: The generic $b \rightarrow c$ semileptonic decay is denoted by $H_{b} \rightarrow H_{c} \ell \bar{\nu}$, where the hadron $H_{b}$ has mass $M$ and momentum $p$, the daughter hadron $H_{c}$ has mass $m$ and momentum $p^{\prime}$, and the charged lepton $\ell$ has mass $m_{\ell}$. The momentum transfer $t=\left(p-p^{\prime}\right)^{2}$ is the invariant mass-squared of the lepton pair (or virtual $W$ ). The polar vector and axial vector flavor-changing currents are denoted by $V^{\mu}=\bar{c} \gamma^{\mu} b$ and $A^{\mu}=\bar{c} \gamma^{\mu} \gamma_{5} b$. Finally, it is convenient to define the kinematic invariants

$$
\begin{aligned}
t_{ \pm} & =(M \pm m)^{2} \\
k & =M \sqrt{\frac{\mathbf{p}^{2}}{t}} \equiv \sqrt{\frac{\left(t_{+}-t\right)\left(t_{-}-t\right)}{4 t}},
\end{aligned}
$$

where $\mathbf{p}$ is the three-momentum of $H_{c}$ in the rest frame of $H_{b}$. Note that the identity of the decaying quark does not enter into the expressions below except through hadron masses, so

the same expressions apply to such decays as $\bar{B} \rightarrow \pi \ell \bar{\nu}, D \rightarrow \bar{K}^{*} \ell \nu$, and so on; only the spins of the hadrons are relevant. 
The inclusion of charged lepton masses brings into the differential widths a new set of helicity amplitudes, and thus a new laboratory for studying the strong interaction. The contributions of such terms is suppressed by a factor of $m_{\ell}^{2}$, and arises through a virtual $W$ with the quantum numbers of a scalar, i.e., a timelike polarization. Angular momentum conservation forbids the decay of such a state to a right-handed antineutrino and a left-handed massless charged lepton, so such decays are necessarily accompanied by a helicity-suppression factor $m_{\ell}^{2}$. Although the detection of lepton mass-dependent effects is presently beyond the means of current experiments, the gradual accumulation of statistics may eventually make such effects discernible in decays like $\bar{B} \rightarrow D^{(*)} \tau \bar{\nu}$ or $\bar{B} \rightarrow \pi \tau \bar{\nu}$.

Finally, it should be noted that the differential widths $d \Gamma / d t$ presented below have already been integrated in lepton energy, or equivalently over angles of final-state particles. This reflects only the current thrust of experiment and does not indicate a limitation of the dispersive method described below. Indeed, were the statistics available, it would be interesting to consider the double differential decay distribution $d^{2} \Gamma / d E_{\ell} d t$, for then one could probe the parity-violating interference terms between vector and axial vector weak currents. In terms of the QCD side of the dispersion relations described below, one would also need to compute $V$-A correlators, in addition to $V-V$ and $A-A$.

\section{A. $\bar{B} \rightarrow D \ell \bar{\nu}$}

The hadronic matrix element governing the rate of $\bar{B} \rightarrow D \ell \bar{\nu}$ may be described by form factors

$$
\left\langle D\left(p^{\prime}\right)\left|V^{\mu}\right| \bar{B}(p)\right\rangle=f_{+}\left(p+p^{\prime}\right)^{\mu}+f_{-}\left(p-p^{\prime}\right)^{\mu}
$$

that enter the differential rate as

$$
\frac{d \Gamma}{d t}=\frac{G_{F}^{2}\left|V_{c b}\right|^{2}}{192 \pi^{3} M^{3}} \frac{k}{t^{\frac{5}{2}}}\left(t-m_{\ell}^{2}\right)^{2}\left[4 k^{2} t\left(2 t+m_{\ell}^{2}\right)\left|f_{+}\right|^{2}+3 m_{\ell}^{2}\left|f_{0}\right|^{2}\right],
$$

where

$$
f_{0}(t) \equiv\left(M^{2}-m^{2}\right) f_{+}(t)+t f_{-}(t)
$$




\section{B. $\bar{B} \rightarrow D^{*} \ell \bar{\nu}$}

The matrix elements for $\bar{B} \rightarrow D^{*} \ell \bar{\nu}$ depend on four form factors,

$$
\begin{aligned}
& \left\langle D^{*}\left(p^{\prime}, \epsilon\right)\left|V^{\mu}\right| \bar{B}(p)\right\rangle=i g \epsilon^{\mu \alpha \beta \gamma} \epsilon_{\alpha}^{*} p_{\beta}^{\prime} p_{\gamma}, \\
& \left\langle D^{*}\left(p^{\prime}, \epsilon\right)\left|A^{\mu}\right| \bar{B}(p)\right\rangle=f \epsilon^{* \mu}+\left(\epsilon^{*} \cdot p\right)\left[a_{+}\left(p+p^{\prime}\right)^{\mu}+a_{-}\left(p-p^{\prime}\right)^{\mu}\right],
\end{aligned}
$$

that enter the differential rate in the combinations

$$
\begin{aligned}
\frac{d \Gamma}{d t}= & \frac{G_{F}^{2}\left|V_{c b}\right|^{2}}{192 \pi^{3} M^{3}} \frac{k}{t^{\frac{5}{2}}}\left(t-m_{\ell}^{2}\right)^{2} \\
& \times\left\{\left(2 t+m_{\ell}^{2}\right)\left[2 t|f|^{2}+\left|\mathcal{F}_{1}\right|^{2}+2 k^{2} t^{2}|g|^{2}\right]+3 m_{\ell}^{2} k^{2} t\left|\mathcal{F}_{2}\right|^{2}\right\}
\end{aligned}
$$

where

$$
\begin{aligned}
& \mathcal{F}_{1}(t) \equiv \frac{1}{m}\left[2 k^{2} t a_{+}(t)-\frac{1}{2}\left(t-M^{2}+m^{2}\right) f(t)\right] \\
& \mathcal{F}_{2}(t) \equiv \frac{1}{m}\left[f(t)+\left(M^{2}-m^{2}\right) a_{+}(t)+t a_{-}(t)\right]
\end{aligned}
$$

\section{C. $\Lambda_{b} \rightarrow \Lambda_{c} \ell \bar{\nu}$}

This decay can be described by six form factors, defined by

$$
\begin{aligned}
& \left\langle\Lambda_{c}\left(p^{\prime}\right)\left|V^{\mu}\right| \Lambda_{b}(p)\right\rangle=\bar{u}_{c}\left(p^{\prime}\right)\left[F_{1} \gamma^{\mu}+F_{2} v^{\mu}+F_{3} v^{\prime \mu}\right] u_{b}(p), \\
& \left\langle\Lambda_{c}\left(p^{\prime}\right)\left|A^{\mu}\right| \Lambda_{b}(p)\right\rangle=\bar{u}_{c}\left(p^{\prime}\right)\left[G_{1} \gamma^{\mu}+G_{2} v^{\mu}+G_{3} v^{\prime \mu}\right] \gamma_{5} u_{b}(p),
\end{aligned}
$$

with $v=p / M_{\Lambda_{b}}$ and $v^{\prime}=p^{\prime} / M_{\Lambda_{c}}$. This gives

$$
\begin{aligned}
\frac{d \Gamma}{d t}= & \frac{G_{F}^{2}\left|V_{c b}\right|^{2}}{192 \pi^{3} M^{3}} \frac{k}{t^{\frac{5}{2}}}\left(t-m_{\ell}^{2}\right)^{2} \\
& \times\left\{\left(t_{-}-t\right)\left(2 t+m_{\ell}^{2}\right)\left[2 t\left|F_{1}\right|^{2}+\left|H_{V}\right|^{2}\right]+3 m_{\ell}^{2}\left(t_{+}-t\right)\left|F_{0}\right|^{2}\right. \\
& \left.+\left(t_{+}-t\right)\left(2 t+m_{\ell}^{2}\right)\left[2 t\left|G_{1}\right|^{2}+\left|H_{A}\right|^{2}\right]+3 m_{\ell}^{2}\left(t_{-}-t\right)\left|G_{0}\right|^{2}\right\},
\end{aligned}
$$

where 


$$
\begin{aligned}
& H_{V}(t)=(M+m) F_{1}+\frac{1}{2}\left(t_{+}-t\right)\left(\frac{F_{2}}{M}+\frac{F_{3}}{m}\right), \\
& H_{A}(t)=(M-m) G_{1}-\frac{1}{2}\left(t_{-}-t\right)\left(\frac{G_{2}}{M}+\frac{G_{3}}{m}\right), \\
& F_{0}(t)=(M-m) F_{1}+\frac{1}{2 M}\left(t+M^{2}-m^{2}\right) F_{2}-\frac{1}{2 m}\left(t-M^{2}+m^{2}\right) F_{3}, \\
& G_{0}(t)=(M+m) G_{1}-\frac{1}{2 M}\left(t+M^{2}-m^{2}\right) G_{2}+\frac{1}{2 m}\left(t-M^{2}+m^{2}\right) G_{3} .
\end{aligned}
$$

\section{REVIEW OF THE DISPERSIVE APPROACH}

Constraints on a generic $H_{b} \rightarrow H_{c} \ell \bar{\nu}$ form factor $F(t)$ are obtained by noting that the amplitude for production of $H_{b} \bar{H}_{c}$ from a virtual $W$ boson is determined by the analytic continuation of $F(t)$ from the semileptonic region of momentum-transfer $m_{\ell}^{2} \leq t \leq t_{-}$to the pair-production region $t_{+} \leq t$. The idea of the dispersion relation is to constrain $F(t)$ in the pair-production region using perturbative QCD, then use analyticity to translate that constraint into one valid in the semileptonic region [19]. A detailed derivation can be found in [7,8, [1]; here we merely outline the essential elements.

In QCD, the two-point function' of a flavor-changing current $J=V, A$, or $V-A$

$$
\Pi_{J}^{\mu \nu}(q)=\frac{1}{q^{2}}\left(q^{\mu} q^{\nu}-q^{2} g^{\mu \nu}\right) \Pi_{J}^{T}\left(q^{2}\right)+\frac{q^{\mu} q^{\nu}}{q^{2}} \Pi_{J}^{L}\left(q^{2}\right) \equiv i \int d^{4} x e^{i q x}\left\langle 0\left|\mathrm{~T} J^{\mu}(x) J^{\dagger \nu}(0)\right| 0\right\rangle,
$$

is rendered finite by making one or two subtractions, leading to the dispersion relations

$$
\begin{array}{r}
\chi_{J}^{L}\left(q^{2}\right) \equiv \frac{\partial \Pi_{J}^{L}}{\partial q^{2}}=\frac{1}{\pi} \int_{0}^{\infty} d t \frac{\operatorname{Im} \Pi_{J}^{L}(t)}{\left(t-q^{2}\right)^{2}}, \\
\chi_{J}^{T}\left(q^{2}\right) \equiv \frac{1}{2} \frac{\partial^{2} \Pi_{J}^{T}}{\partial\left(q^{2}\right)^{2}}=\frac{1}{\pi} \int_{0}^{\infty} d t \frac{\operatorname{Im} \Pi_{J}^{T}(t)}{\left(t-q^{2}\right)^{3}} .
\end{array}
$$

The functions $\chi\left(q^{2}\right)$ may be computed reliably in perturbative QCD for values of $q^{2}$ far from the kinematic region where the current $J$ can create resonances: specifically, $\left(m_{b}+m_{c}\right) \Lambda_{\mathrm{QCD}} \ll\left(m_{b}+m_{c}\right)^{2}-q^{2}$. For $b \rightarrow c$, or $u, q^{2}=0$ satisfies this condition.

\footnotetext{
${ }^{1}$ This definition differs slightly from that used in [1], and serves to separate $\Pi^{\mu \nu}$ into manifestly spin-0 and spin-1 pieces. Then the functions $\chi^{T}, \chi^{L}$ defined in (3.2) coincide with $\chi, \chi^{L}$ defined in the previous works.
} 
Inserting a complete set of states $X$ into the two-point function relates the $\Pi_{J}$ to the production rate of hadrons from a virtual $W$,

$$
\operatorname{Im} \Pi_{J}^{T, L}=\frac{1}{2} \sum_{X}(2 \pi)^{4} \delta^{4}\left(q-p_{X}\right)|\langle 0|J| X\rangle|^{2}
$$

where the sum is over all hadronic states $X$ with the same quantum numbers as the current $J$, weighted by phase space. Then, from the dispersion relations (3.2), the perturbatively evaluated $\chi\left(q^{2}\right)$ is equal to the integrated production rate of $W^{*} \rightarrow X$, weighted with a smooth function of momentum transfer squared, $t$. Since the sum is semipositive definite, one may restrict attention to a subset of hadronic states to obtain a strict inequality. In the case of interest, we focus on $X$ being two-particle states of the form $H_{b} \bar{H}_{c}$. This places an upper bound on the form factor $F(t)$ in the pair-production region that takes the form

$$
\frac{1}{\pi \chi^{T}\left(q^{2}\right)} \int_{t_{+}}^{\infty} d t \frac{W(t)|F(t)|^{2}}{\left(t-q^{2}\right)^{3}} \leq 1
$$

from the $\operatorname{Im} \Pi^{T}$ dispersion relations in Eq. (3.2). Here $W(t)$ is a computable function of $t$ that depends on the particular form factor under consideration. A similar result holds for $\Pi^{L}$.

Using analyticity to turn (3.4) into a constraint in the semileptonic region requires that the integrand is analytic below the pair-production threshold $t<t_{+}$. To do this, we introduce a function

$$
z\left(t ; t_{s}\right)=\frac{t_{s}-t}{\left(\sqrt{t_{+}-t}+\sqrt{t_{+}-t_{s}}\right)^{2}}
$$

that is real for $t_{s}<t_{+}$, zero at $t=t_{s}$, and a pure phase for $t \geq t_{+}$. All the poles in the integrand of Eq. (3.4) can be removed by multiplying by various powers of $z\left(t ; t_{s}\right)$, provided the positions $t_{s}$ of the sub-threshold poles in $F(t)$ are known. Each pole has a distinct value of $t_{s}$, and the product $z\left(t ; t_{s 1}\right) z\left(t ; t_{s 2}\right) \cdots$ serves to remove all of them. Such poles arise as the contribution of $B_{c}$ resonances to the form factor $F(t)$, as well as singularities in the kinematic part of the integrand. After determining these positions phenomenologically, the upper bound on $F(t)$ becomes 


$$
\frac{1}{\pi} \int_{t_{+}}^{\infty} d t\left|\frac{d z\left(t ; t_{0}\right)}{d t}\right| \cdot\left|\phi\left(t ; t_{0}\right) P(t) F(t)\right|^{2} \leq 1,
$$

where the weight function $\phi\left(t ; t_{0}\right)$ (known as an outer function in complex analysis) is given by

$$
\phi\left(t ; t_{0}\right)=\tilde{P}(t)\left[\frac{W(t)}{\left|d z\left(t ; t_{0}\right) / d t\right| \chi^{T}\left(q^{2}\right)\left(t-q^{2}\right)^{3}}\right]^{\frac{1}{2}} .
$$

The factor $\tilde{P}(t)$ is a product of $z\left(t ; t_{s}\right)$ 's and $\sqrt{z\left(t ; t_{s}\right)}$ 's, with $t_{s}$ chosen to remove the subthreshold singularities and cuts in the kinematic part of the integrand, while the Blaschke factor $P(t)$ is a product of $z\left(t ; t_{p}\right)$ 's with $t_{p}$ chosen to be the positions of sub-threshold poles in $F(t)$. The functions $\phi\left(t ; t_{0}\right)$ and $\tilde{P}(t)$ also depend on $q^{2}$, which we leave implicit for notational simplicity, while $t_{0}$ is a free parameter to be discussed in Sec. IV.

The quantity $\phi\left(t ; t_{0}\right) P(t) F(t)$ may be expanded in a set of orthonormal functions that are proportional to powers of $z\left(t ; t_{0}\right)$ [see (4.21) below]. The function $z\left(t ; t_{0}\right)$ has a physical interpretation as a natural scale for the variation of $F(t)$ in the semileptonic region [11], and will play an important role throughout this paper. We exhibit all relevant expressions in terms of both variables, $z$ and $t$, in Sec. IV. The result of the expansion in $z\left(t ; t_{0}\right)$ is an expression for $F(t)$ valid even in the semileptonic region,

$$
F(t)=\frac{1}{P(t) \phi\left(t ; t_{0}\right)} \sum_{n=0}^{\infty} a_{n} z\left(t ; t_{0}\right)^{n}
$$

where, as a result of Eq. (3.6), the coefficients $a_{n}$ are unknown constants obeying

$$
\sum_{n=0}^{\infty} a_{n}^{2} \leq 1
$$

For the $b \rightarrow c$ processes that are the main subject of this paper, $z\left(t ; t_{0}\right)$ is no larger than 0.07

for any physical momentum transfer $m_{\ell}^{2} \leq t \leq t_{-}$, and can be made substantially smaller by a judicious choice of $t_{0}$, so the expansion can be truncated after the first two or three terms.

\section{PARAMETERIZATION INGREDIENTS}

Generating a parameterization like (3.8) for a particular form factor requires three ingredients: One needs the perturbative evaluation of $\chi$ derived from the two-point function for a 
current $J$, including the Wilson coefficients of phenomenologically determined condensates. In addition, the functional form of the weighting function $\phi$ must be computed. Finally, the masses of sub-threshold resonances with the same quantum numbers as $J$ must be extracted from experiment or potential models. The function $\phi$ depends on the form factor under consideration, while $\chi$ and $P$ depend only on the current $J$.

\section{A. QCD Evaluation of $\chi$}

In the previous section we observed that it suffices to take $q^{2}=0$ in the computation of $\chi_{J}^{T, L}$ for currents containing a heavy quark. This is convenient since then the perturbative expressions become particularly simple. Corrections to the perturbative result may be included by expressing the two-point function as an operator product expansion (OPE) and including the leading nonperturbative vacuum condensates such as $\left\langle G^{2}\right\rangle$ and $\langle\bar{q} q\rangle$; the total $\chi$ is the sum of the perturbative and condensate terms,

$$
\chi=\chi_{\text {pert }}+\chi_{\text {cond }}
$$

The full perturbative expressions to two loops may be obtained through a lengthy but straightforward $^{2}$ manipulation of results existing in the literature [20,22]. At $q^{2}=0, \chi_{\text {pert }}$ is only a function of the ratio of quark pole masses $u=m_{c} / m_{b}$, and for a vector current $J=V$ is given by

$$
\begin{aligned}
& m_{b}^{2} \chi_{\text {pert }}^{T}(u)= \\
& \frac{1}{32 \pi^{2}\left(1-u^{2}\right)^{5}}\left[\left(1-u^{2}\right)\left(3+4 u-21 u^{2}+40 u^{3}-21 u^{4}+4 u^{5}+3 u^{6}\right)\right.
\end{aligned}
$$

\footnotetext{
${ }^{2}$ We have corrected a number of typographical errors in the perturbative results of Ref. [20] and the Wilson Coefficient results of [21] to ensure compliance with various consistency conditions. We also made several comparisons between references to verify their agreement, once these corrections are made.
} 


$$
\begin{aligned}
& \left.+12 u^{3}\left(2-3 u+2 u^{2}\right) \ln \left(u^{2}\right)\right] \\
& +\frac{\alpha_{s}}{576 \pi^{3}\left(1-u^{2}\right)^{6}} \\
& \quad\left[\left(1-u^{2}\right)^{2}\left(75+360 u-1031 u^{2}+1776 u^{3}-1031 u^{4}+360 u^{5}+75 u^{6}\right)\right. \\
& \quad+4 u\left(1-u^{2}\right)\left(18-99 u+732 u^{2}-1010 u^{3}+732 u^{4}-99 u^{5}+18 u^{6}\right) \ln \left(u^{2}\right) \\
& +4 u^{3}\left(108-324 u+648 u^{2}-456 u^{3}+132 u^{4}+59 u^{5}-12 u^{6}-9 u^{7}\right) \ln ^{2}\left(u^{2}\right) \\
& \left.+8\left(1-u^{2}\right)^{3}\left(9+12 u-32 u^{2}+12 u^{3}+9 u^{4}\right) \operatorname{Li}_{2}\left(1-u^{2}\right)\right], \\
& \chi_{\text {pert }}^{L}(u)= \\
& \frac{1}{8 \pi^{2}\left(1-u^{2}\right)^{3}}\left[\left(1-u^{2}\right)\left(1+u+u^{2}\right)\left(1-4 u+u^{2}\right)-6 u^{3} \ln \left(u^{2}\right)\right] \\
& +\frac{\alpha_{s}}{48 \pi^{3}\left(1-u^{2}\right)^{4}}\left[\left(1-u^{2}\right)^{2}\left(1-36 u-22 u^{2}-36 u^{3}+u^{4}\right)\right. \\
& \quad-2 u\left(1-u^{2}\right)\left(9+4 u+66 u^{2}+4 u^{3}+9 u^{4}\right) \ln \left(u^{2}\right) \\
& \quad-4 u^{3}\left(9+18 u^{2}-2 u^{3}-3 u^{4}+u^{5}\right) \ln ^{2}\left(u^{2}\right)+8\left(1-u^{2}\right)^{3}\left(1-3 u+u^{2}\right) \\
& \left.\quad \times \operatorname{Li}_{2}\left(1-u^{2}\right)\right],
\end{aligned}
$$

where the dilogarithm is defined by

$$
\operatorname{Li}_{2}(z) \equiv-\int_{0}^{z} d z^{\prime} \frac{\ln \left(1-z^{\prime}\right)}{z^{\prime}}
$$

Expressions for an axial current $J=A$ are obtained from (4.2), (4.3) by replacing $u \rightarrow-u$.

It has been pointed out [23] that non-analytic quark mass dependence, such as in the perturbative results presented above, indicates the inclusion of some infrared effects into the Wilson Coefficients (WC's), in conflict with the usual interpretation of the OPE as a separation into short- and long-distance effects. A formal analysis reshuffles the WC's in front of each nonperturbative condensate. However, since our analysis requires only the numerical sum of such effects, the total result should be the same in either form.

The leading nonperturbative corrections are supplied by the condensates of dimension less than five, namely the gluon and quark condensates. For a heavy $b$ quark decaying into a quark $q$ of arbitrary mass through a vector current, the contributions from the condensates, derived using Refs. [21,24] and evaluated at $q^{2}=0$, read 


$$
\begin{aligned}
& \chi_{\text {cond }}^{T}(u)= \\
& -\langle\bar{q} q\rangle \frac{\left(2-3 u+2 u^{2}\right)}{2 m_{b}^{5}\left(1-u^{2}\right)^{5}} \\
& +\left\langle\frac{\alpha_{s}}{\pi} G^{2}\right\rangle\left\{\frac{-1}{24 m_{b}^{6}\left(1-u^{2}\right)^{7}}\right. \\
& \times\left[\left(1-u^{2}\right)\left(2-104 u+148 u^{2}-270 u^{3}+145 u^{4}-104 u^{5}+5 u^{6}-2 u^{7}\right)\right. \\
& \left.\left.\chi_{\text {cond }}^{L}(u)=\quad-12 u \ln \left(u^{2}\right)\left(3-5 u+17 u^{2}-15 u^{3}+17 u^{4}-5 u^{5}+3 u^{6}\right)\right]\right\}, \\
& +\langle\bar{q} q\rangle \frac{1}{m_{b}^{3}\left(1-u^{2}\right)^{3}}\left[\left(1-u^{2}\right)\left(1-21 u+10 u^{2}-20 u^{3}+u^{4}-u^{5}\right)\right. \\
& +\left\langle\frac{\alpha_{s}}{\pi} G^{2}\right\rangle\left\{\frac{1}{12 m_{b}^{4}\left(1-u^{2}\right)^{5}}\left[3 u \ln \left(u^{2}\right)\left(3-2 u+8 u^{2}-2 u^{3}+3 u^{4}\right)\right]\right\} .
\end{aligned}
$$

These expressions superficially appear to diverge in the limit $u \rightarrow 1$. However, in this limit the $\left\langle m_{q} \bar{q} q\right\rangle$ condensate obeys the relation [25]

$$
\left\langle m_{q} \bar{q} q\right\rangle=-\frac{1}{12}\left\langle\frac{\alpha_{s}}{\pi} G^{2}\right\rangle+O\left(\frac{1}{m_{q}^{2}}, \alpha_{s}\right),
$$

and Eqs. (4.5), (4.6) simplify to

$$
\begin{aligned}
\chi_{\text {cond }}^{T}(u)= & \left\langle\frac{\alpha_{s}}{\pi} G^{2}\right\rangle \frac{1}{24\left(1-u^{2}\right)^{7} m_{b}^{6} u} \\
& \times\left\{\left(1-u^{2}\right)\left(2-5 u+104 u^{2}-145 u^{3}+268 u^{4}-145 u^{5}+104 u^{6}-5 u^{7}+2 u^{8}\right)\right. \\
& \left.+12 u^{2} \ln \left(u^{2}\right)\left(3-5 u+17 u^{2}-15 u^{3}+17 u^{4}-5 u^{5}+3 u^{6}\right)\right\}, \\
\chi_{\text {cond }}^{L}(u)=- & \left\langle\frac{\alpha_{s}}{\pi} G^{2}\right\rangle \frac{1}{12\left(1-u^{2}\right)^{5} m_{b}^{4} u}\left\{\left(1-u^{2}\right)\left(1-u+20 u^{2}-10 u^{3}+20 u^{4}-u^{5}+u^{6}\right)\right. \\
& \left.+3 u^{2} \ln \left(u^{2}\right)\left(3-2 u+8 u^{2}-2 u^{3}+3 u^{4}\right)\right\},
\end{aligned}
$$

The expressions (4.8), (4.9) are applicable to $b \rightarrow c$ decays, while (4.5), (4.6) are best suited to $b \rightarrow u$ or $c \rightarrow s$ transitions. Expressions for an axial current are obtained by replacing $u \rightarrow-u$. The limiting values for $u \rightarrow 0$ are 


$$
\begin{aligned}
m_{b}^{2} \chi^{T}(0) & =\frac{3}{32 \pi^{2}}+\frac{\alpha_{s}}{192 \pi^{3}}\left(25+4 \pi^{2}\right)-\frac{1}{m_{b}^{3}}\langle\bar{q} q\rangle-\frac{1}{12 m_{b}^{4}}\left\langle\frac{\alpha_{s}}{\pi} G^{2}\right\rangle, \\
\chi^{L}(0) & =\frac{1}{8 \pi^{2}}+\frac{\alpha_{s}}{144 \pi^{3}}\left(3+4 \pi^{2}\right)+\frac{1}{m_{b}^{3}}\langle\bar{q} q\rangle+\frac{1}{12 m_{b}^{4}}\left\langle\frac{\alpha_{s}}{\pi} G^{2}\right\rangle,
\end{aligned}
$$

while for $u \rightarrow 1$,

$$
\begin{aligned}
m_{b}^{2} \chi^{T}(+1) & =\frac{1}{20 \pi^{2}}+\frac{41 \alpha_{s}}{162 \pi^{3}}-\frac{1}{210 m_{b}^{4}}\left\langle\frac{\alpha_{s}}{\pi} G^{2}\right\rangle \\
m_{b}^{2} \chi^{T}(-1) & =\frac{1}{40 \pi^{2}}+\frac{689 \alpha_{s}}{6480 \pi^{3}}-\frac{1}{140 m_{b}^{4}}\left\langle\frac{\alpha_{s}}{\pi} G^{2}\right\rangle, \\
\chi^{L}(+1) & =0 \\
\chi^{L}(-1) & =\frac{1}{4 \pi^{2}}+\frac{7 \alpha_{s}}{12 \pi^{3}}+\frac{1}{60 m_{b}^{4}}\left\langle\frac{\alpha_{s}}{\pi} G^{2}\right\rangle .
\end{aligned}
$$

For $b \rightarrow c$, using pole mass values such that $u=0.33$, we have

$$
\begin{aligned}
m_{b}^{2} \chi^{T}(+0.33) & =9.659 \cdot 10^{-3}\left[1+1.42 \alpha_{s}-4.8 \cdot 10^{-4}\left(\frac{4.9 \mathrm{GeV}}{m_{b}}\right)^{4}\left\langle\frac{\left(\alpha_{s} G^{2} / \pi\right)}{0.02 \mathrm{GeV}^{4}}\right\rangle\right], \\
m_{b}^{2} \chi^{T}(-0.33) & =5.709 \cdot 10^{-3}\left[1+1.32 \alpha_{s}-6.8 \cdot 10^{-4}\left(\frac{4.9 \mathrm{GeV}}{m_{b}}\right)^{4}\left\langle\frac{\left(\alpha_{s} G^{2} / \pi\right)}{0.02 \mathrm{GeV}^{4}}\right\rangle\right], \\
\chi^{L}(+0.33) & =3.713 \cdot 10^{-3}\left[1+1.37 \alpha_{s}-5.3 \cdot 10^{-4}\left(\frac{4.9 \mathrm{GeV}}{m_{b}}\right)^{4}\left\langle\frac{\left(\alpha_{s} G^{2} / \pi\right)}{0.02 \mathrm{GeV}^{4}}\right\rangle\right], \\
\chi^{L}(-0.33) & =2.162 \cdot 10^{-3}\left[1+0.64 \alpha_{s}+2.2 \cdot 10^{-4}\left(\frac{4.9 \mathrm{GeV}}{m_{b}}\right)^{4}\left\langle\frac{\left(\alpha_{s} G^{2} / \pi\right)}{0.02 \mathrm{GeV}^{4}}\right\rangle\right],
\end{aligned}
$$

where the central value $<\frac{\alpha_{s}}{\pi} G^{2}>=0.02 \mathrm{GeV}^{4}$ is taken from Ref. [26], and the pole mass value $m_{b}=4.9 \mathrm{GeV}$ is from [27]. We also use $\alpha_{s}\left(m_{b}\right)=0.22$ [27], and since the coefficient of the gluon condensate is tiny, we ignore it in our numerical analysis for $b \rightarrow c$.

\section{B. Weighting functions $\phi$}

To obtain the general form of the weighting functions $\phi$ defined in Eq. (3.6), first observe that the quantities $\Pi^{L}, \Pi^{T}$ are, respectively, the $\mu=\nu=0$ and $\mu=\nu=1,2$, or 3 components of $\Pi^{\mu \nu}$ evaluated in the center of mass frame, $q^{\mu}=(\sqrt{t}, \mathbf{0})$. Then the generic expression for the contribution of a particular form factor $F(t)$ to the polarization tensor may be denoted by 


$$
\begin{aligned}
\operatorname{Im} \Pi^{T} \geq \frac{n_{I}}{K \pi}\left(t-t_{+}\right)^{\frac{a}{2}}\left(t-t_{-}\right)^{\frac{b}{2}} t^{-c}|F(t)|^{2} \theta\left(t-t_{+}\right), \\
\operatorname{Im} \Pi^{L} \geq \frac{n_{I}}{K \pi}\left(t-t_{+}\right)^{\frac{a}{2}}\left(t-t_{-}\right)^{\frac{b}{2}} t^{-(c+1)}|F(t)|^{2} \theta\left(t-t_{+}\right),
\end{aligned}
$$

where $K, a, b$, and $c$ are integers determined by the form factor $F(t)$, and $n_{I}$ is an isospin Clebsch-Gordan factor, which is $2,3 / 2$, and 1 for $\bar{B} \rightarrow D^{(*)}, B \rightarrow \pi$, and $\Lambda_{b} \rightarrow \Lambda_{c}$ transitions, respectively. Also, let $\chi=\chi^{T}$ or $\chi^{L}$ denote the generic QCD function appropriate to the quantum numbers of the form factor. The expressions for the weighting functions are readily derived from Eq. (4.13), and are given by

$$
\begin{aligned}
\phi_{i}\left(t ; t_{0}\right)= & \sqrt{\frac{n_{I}}{K \pi \chi}}\left(\frac{t_{+}-t}{t_{+}-t_{0}}\right)^{\frac{1}{4}}\left(\sqrt{t_{+}-t}+\sqrt{t_{+}-t_{0}}\right)\left(t_{+}-t\right)^{\frac{a}{4}} \\
& \times\left(\sqrt{t_{+}-t}+\sqrt{t_{+}-t_{-}}\right)^{\frac{b}{2}}\left(\sqrt{t_{+}-t}+\sqrt{t_{+}}\right)^{-(c+3)} .
\end{aligned}
$$

The values of the parameters $K, a, b, c$ for each form factor, as well as the relevant $\chi$, are given in Table 1 for $\bar{B} \rightarrow D^{(*)}$ transitions, and in Table 2 for $\Lambda_{b} \rightarrow \Lambda_{c}$. Although (4.14) assumes $q^{2}=0$, it is easy to generalize to arbitrary $Q^{2} \equiv-q^{2}$ : Simply evaluate the perturbative functions $\chi\left(Q^{2}\right)$ at the given value, and multiply $\phi$ by

$$
\left(\frac{\sqrt{t_{+}-t}+\sqrt{t_{+}}}{\sqrt{t_{+}-t}+\sqrt{t_{+}+Q^{2}}}\right)^{d},
$$

with $d=3$ if the form factor involves $\chi^{T}$ and $d=2$ if it involves $\chi^{L}$.

\footnotetext{
${ }^{3}$ In terms of previous notations, one finds for the meson form factors considered in Refs. [7,8] $K=2^{p} \kappa^{-1} / \pi \chi M^{2}, a=b=p, c=s+p-3$, whereas in Ref. [11 one finds $K=3 \cdot 2^{s}, a=b=w$, and $c=p$. For the baryon form factors considered in [7], the relation is given by by $a=2 p+1$, $b=3-2 p, c=s+1$, and $K=2 \kappa^{-1} / \pi \chi M^{2}$ or $K=2 \kappa^{-1} / \pi \chi^{L}$, depending upon whether the form factor appears with $\chi$ or $\chi^{L}$.
} 


\begin{tabular}{|r|c|c|c|c|c|}
\hline$F_{i}$ & $K$ & $\chi$ & $a$ & $b$ & $c$ \\
\hline$f_{+}$ & 48 & $\chi^{T}(+u)$ & 3 & 3 & 2 \\
$f_{0}$ & 16 & $\chi^{L}(+u)$ & 1 & 1 & 1 \\
\hline$f$ & 24 & $\chi^{T}(-u)$ & 1 & 1 & 1 \\
$\mathcal{F}_{1}$ & 48 & $\chi^{T}(-u)$ & 1 & 1 & 2 \\
$g$ & 96 & $\chi^{T}(+u)$ & 3 & 3 & 1 \\
$\mathcal{F}_{2}$ & 64 & $\chi^{L}(-u)$ & 3 & 3 & 1 \\
\hline
\end{tabular}

Table 1. Factors entering Eq. (4.14) or (4.23) for the meson form factors $F_{i}$ in $\bar{B} \rightarrow D^{(*)}$.

\begin{tabular}{|c|r|c|c|c|c|}
\hline$F_{i}$ & \multicolumn{1}{|c|}{$K$} & $\chi$ & $a$ & $b$ & $c$ \\
\hline$F_{0}$ & 8 & $\chi^{L}(+u)$ & 3 & 1 & 1 \\
$F_{1}$ & 12 & $\chi^{T}(+u)$ & 1 & 3 & 1 \\
$H_{V}$ & 24 & $\chi^{T}(+u)$ & 1 & 3 & 2 \\
$G_{0}$ & 8 & $\chi^{L}(-u)$ & 1 & 3 & 1 \\
$G_{1}$ & 12 & $\chi^{T}(-u)$ & 3 & 1 & 1 \\
$H_{A}$ & 24 & $\chi^{T}(-u)$ & 3 & 1 & 2 \\
\hline
\end{tabular}

Table 2. Factors entering Eq. (4.14) or (4.23) for the baryon form factors $F_{i}$.

While the momentum-transfer variable $t=\left(p-p^{\prime}\right)^{2}$ is useful for heavy-to-light decays and has an obvious physical meaning, it is often more convenient when dealing with heavyto-heavy transitions such as $b \rightarrow c$ to use a kinematic variable that helps disentangle longdistance physics from the heavy quark scale. One such variable is

$$
w \equiv v \cdot v^{\prime}=\frac{M^{2}+m^{2}-t}{2 M m} .
$$

In the $b$ rest frame, $w$ depends only on the energy transfer to the light degrees of freedom, in units of $\Lambda_{\mathrm{QCD}}$. It is due to this property that $b \rightarrow c$ form factors are related to each other in the heavy quark limit at equal values of $w$. In the semileptonic region, the variable $z\left(t ; t_{0}\right)$ has the same physical property, so form factors will be related at equal values of $z$ 
as well. We can demonstrate this by expressing $z$ as a function of $w$ without reference to heavy meson masses,

$$
z(w ; N)=\frac{\sqrt{1+w}-\sqrt{2 N}}{\sqrt{1+w}+\sqrt{2 N}},
$$

where $N$ is a free parameter related to $t_{0}$ by

$$
N=\frac{t_{+}-t_{0}}{t_{+}-t_{-}}
$$

so that $z(w ; N)$ vanishes at $w=2 N-1$. With $t, t_{0}$ related to $w, N$ by Eqs. (4.16) and (4.18), Eq. (4.17) is simply a rewriting of Eq. (3.5), $z\left(t ; t_{0}\right)=z(w ; N)$. The advantage in using $z(w ; N)$ for $b \rightarrow c$ transitions is that its definition is process-independent.

For the semileptonic decay $H_{b} \rightarrow H_{c} \ell \bar{\nu}$, the limiting values of $z$ are given by $m_{\ell}^{2} \leq t \leq t_{-}$, or

$$
z_{\min }=-\left(\frac{\sqrt{N}-1}{\sqrt{N}+1}\right)
$$

and

$$
z_{\max }=\frac{\sqrt{(1+r)^{2}-\delta^{2}}-2 \sqrt{N r}}{\sqrt{(1+r)^{2}-\delta^{2}}+2 \sqrt{N r}} .
$$

where $r=m / M$ and $\delta=m_{\ell} / M$.

The dispersion relation (3.6), written now entirely in terms of $z$, reads

$$
\frac{1}{2 \pi i} \int_{C} \frac{d z}{z}|\phi(z) P(z) F(z)|^{2} \leq 1
$$

where $C$ is the unit circle in the complex $z$ plane, the Blaschke factor for a pole at $z_{p} \equiv$ $z\left(t_{p} ; t_{0}\right)$ (which is real for sub-threshold resonance masses) is

$$
z\left(t ; t_{p}\right)=\frac{z-z_{p}}{1-z z_{p}}
$$

for $z=z\left(t ; t_{0}\right)$ and any $t_{0}$, with $P(z)$ being the product of all such factors, and the weighting functions (4.14) are given by 


$$
\begin{aligned}
\phi(z ; N)= & M^{\frac{1}{2}(a+b)-(c+2)} \sqrt{\frac{n_{I}}{K \pi \chi}} 2^{\frac{1}{2}(a+b)+2} N^{\frac{a}{4}+\frac{1}{2}} r^{\frac{1}{4}(a+b)+\frac{1}{2}}(1+z)^{\frac{1}{2}(a+1)}(1-z)^{c+\frac{1}{2}(3-a-b)} \\
& \times[(\sqrt{N}-1) z+(\sqrt{N}+1)]^{\frac{b}{2}}[(1+r)(1-z)+2 \sqrt{N r}(1+z)]^{-(c+3)}
\end{aligned}
$$

Finally, evaluation of $\phi(z ; N)$ at nonzero values of $Q^{2}=-q^{2}$ is accomplished by multiplying (4.23) by

$$
\left(\frac{(1+r)(1-z)+2 \sqrt{N r}(1+z)}{\sqrt{(1+r)^{2}+Q^{2} / M^{2}}(1-z)+2 \sqrt{N r}(1+z)}\right)^{d},
$$

where $d$ is defined as in Eq. (4.15).

\section{Sub-threshold Resonances and $P(t)$}

The Blaschke factor $P(t)$ for a form factor describing $H_{b} \rightarrow H_{c} \ell \bar{\nu}$ depends on the masses of $B_{c}$ resonances below the $H_{b} \bar{H}_{c}$ pair-production threshold. The Blaschke factors are simply products of $z\left(t ; t_{s}\right)$ with $t_{s}$ evaluated at the invariant mass-squared $t_{s} \rightarrow t_{p}$ of each such resonance with the same spin-parity as the current $J$,

$$
P(t)=\prod_{p} z\left(t ; t_{p}\right)
$$

For $b \rightarrow c$ transition from factors, the masses of the relevant $B_{c}$-type resonances can be accurately estimated from potential models 228,29. We compile in Table 3 the masses computed in Ref. 29.

\begin{tabular}{|r|r|}
\hline Type & Masses $(\mathrm{GeV})$ \\
\hline Vector & $6.337,6.899,7.012,7.280$ \\
& $7.350,7.594,7.646,7.872,7.913$ \\
\hline Axial vector & $6.730,6.736,7.135,7.142$ \\
& $7.470,7.470,7.757,7.757$ \\
\hline Scalar & $6.700,7.108,7.470,7.757$ \\
\hline Pseudoscalar & $6.264,6.856,7.244,7.562,7.844$ \\
\hline
\end{tabular}

Table 3. Calculated $B_{c}$ pole masses used in this work. 
For heavy-to-light form factors there is no formal limit in which the light quark becomes nonrelativistic, and potential model calculations are less reliable. This is not a problem for $B \rightarrow \pi$, where the only sub-threshold resonance, the $B^{*}$, is experimentally observed, but for decays to other light states such as $B \rightarrow \rho$, the presence and masses of additional subthreshold resonances must be taken from models. Once these uncertainties are accounted for, simple parameterizations should be reliable. For example, the model of [30] indicates that the form factor $f$ for $B \rightarrow \rho$ has only one narrow sub-threshold pole. Indeed, this pole appears to have been observed (mixed with others) by ALEPH, DELPHI, and OPAL [see [27] for analysis and references on the " $B_{J}^{*}(5732)$ "]. Even accounting for significant uncertainties in its mass, this leads to an accurate parameterization using the overall normalization and two unknown coefficients. It is important to estimate the uncertainties from model-dependent poles on a case by case basis 14 .

This applies as well to sub-threshold branch cuts due to multi-particle states and anomalous thresholds. A model-dependent analysis [7,8] suggests these are negligible for $b \rightarrow c$ transitions. Qualitatively, this result comes about because cuts are a much less severe form of non-analytic behavior than poles. Whether cuts continue to be unimportant for $B \rightarrow \rho$ transitions requires a more detailed analysis.

\section{ADDITIONAL STATES}

The effects of higher states in the dispersion relation depend on the flavor of the $b \rightarrow q$ current under consideration. Henceforth, we specialize to $b \rightarrow c$ transitions, for which HQET is most useful.

\section{A. Contributions to the Dispersion Relation}

We have observed that contributions to the original dispersion relation [see Eq. (3.3)] are semipositive definite, so each additional state coupling to the vacuum through the current $J$ serves to further saturate the bound supplied by the QCD parton-level calculation. The 
inclusion of only a single two-particle $\bar{B} \bar{D}$ or $\bar{B} \bar{D}^{*}$ state in obtaining these bounds is relatively weak, since such exclusive states account for only a small portion of the inclusive total. In general, the hadronic side includes also $B_{c}$ resonances, a continuum of states like $\bar{B} \bar{D} \pi \pi$, and so on. While it is desirable to include as many of these states as possible, it is not clear how to include them in a model-independent fashion; the chief exceptions are twoparticle states related to one another via heavy quark spin symmetry, namely the four states $\bar{B}^{(*)} \bar{D}^{(*)}$. While form factors for transitions such as $\bar{B}^{*} \rightarrow D^{(*)}$ are not physically accessible through semileptonic decays, their normalization is nonetheless known via HQET, allowing an additional strengthening of the dispersive bounds.

The contributions (4.13) of these states to the dispersion relations are of the form

$$
\operatorname{Im} \Pi(t) \geq \sum_{i} \kappa_{i}(t)\left|F_{i}(t)\right|^{2}
$$

where the sum is over all helicity amplitudes $F_{i}$ arising from pair production of $\bar{B}^{(*)} \bar{D}^{(*)}$. The weight functions $\phi$ are readily obtained from the kinematic prefactors $\kappa_{i}(t)$, while the relation between the helicity amplitudes and the original form factors may be obtained by choosing definite polarizations of the $\bar{B}^{*}, \bar{D}^{*}$, and the virtual $W$.

For $\bar{B}^{*} \rightarrow D$ transitions, the form factors are defined by

$$
\begin{aligned}
\left\langle D\left(p^{\prime}\right)\left|V^{\mu}\right| \bar{B}^{*}(p, \epsilon)\right\rangle & =i \hat{g} \epsilon^{\mu \alpha \beta \gamma} \epsilon_{\alpha} p_{\beta} p_{\gamma}^{\prime} \\
\left\langle D^{*}\left(p^{\prime}\right)\left|A^{\mu}\right| \bar{B}^{*}(p, \epsilon)\right\rangle & =\hat{f} \epsilon^{\mu}+\left(\epsilon \cdot p^{\prime}\right)\left[\hat{a}_{+}\left(p^{\prime}+p\right)^{\mu}+\hat{a}_{-}\left(p^{\prime}-p\right)^{\mu}\right] .
\end{aligned}
$$

The functions $\phi$ for $\bar{B}^{*} \rightarrow D$ transitions are identical to those for $\bar{B} \rightarrow D^{*}$, with the simple replacement $M \leftrightarrow m$. The helicity amplitudes possess factors of $M$ and $m$, and need not be invariant under this exchange. This is true in particular for $\mathcal{F}_{1}$ and $\mathcal{F}_{2}$; here we find that [compare (2.7)]

$$
\begin{aligned}
& \hat{\mathcal{F}}_{1}(t) \equiv \frac{1}{M}\left[2 k^{2} t \hat{a}_{+}(t)-\frac{1}{2}\left(t-m^{2}+M^{2}\right) \hat{f}(t)\right] \\
& \hat{\mathcal{F}}_{2}(t) \equiv \frac{1}{M}\left[\hat{f}(t)-\left(M^{2}-m^{2}\right) \hat{a}_{+}(t)+t \hat{a}_{-}(t)\right]
\end{aligned}
$$


The transition $\bar{B}^{*} \rightarrow D^{*}$ possesses 10 independent vector current form factors, which we define as follows 31:

$$
\begin{aligned}
\frac{1}{\sqrt{M m}}\left\langle D^{*}\left(\epsilon_{D}, v^{\prime}\right)\left|V^{\mu}\right| \bar{B}^{*}\left(\epsilon_{B}, v\right)\right\rangle= & f_{4}\left(\epsilon_{B} \cdot \epsilon_{D}^{*}\right) v^{\prime \mu}+f_{5}\left(\epsilon_{B} \cdot \epsilon_{D}^{*}\right) v^{\mu} \\
& +f_{6}\left(v^{\prime} \cdot \epsilon_{B}\right)\left(v \cdot \epsilon_{D}^{*}\right) v^{\mu}+f_{7}\left(v^{\prime} \cdot \epsilon_{B}\right)\left(v \cdot \epsilon_{D}^{*}\right) v^{\prime \mu} \\
& +f_{8}\left(v \cdot \epsilon_{D}^{*}\right) \epsilon_{B}^{\mu}+f_{9}\left(v^{\prime} \cdot \epsilon_{B}\right) \epsilon_{D}^{* \mu}, \\
\frac{1}{\sqrt{M m}}\left\langle D^{*}\left(\epsilon_{D}, v^{\prime}\right)\left|A^{\mu}\right| \bar{B}^{*}\left(\epsilon_{B}, v\right)\right\rangle= & +i f_{10} \epsilon^{\mu}{ }_{\alpha \beta \gamma} \epsilon_{B}^{\alpha} \epsilon_{D}^{* \beta} v^{\prime \gamma}+i f_{11} \epsilon_{\alpha \beta \gamma}^{\mu} \epsilon_{B}^{\alpha} \epsilon_{D}^{* \beta} v^{\gamma} \\
& +i f_{12}\left[\left(v^{\prime} \cdot \epsilon_{B}\right) \epsilon_{\alpha \beta \gamma}^{\mu} \epsilon_{D}^{* \alpha} v^{\prime \beta} v^{\gamma}+\epsilon_{\alpha \beta \gamma \delta} \epsilon_{B}^{\alpha} \epsilon_{D}^{* \beta} v^{\prime \gamma} v^{\delta} v^{\prime \mu}\right] \\
& +i f_{13}\left[\left(v \cdot \epsilon_{D}\right) \epsilon^{\mu}{ }_{\alpha \beta \gamma} \epsilon_{B}^{* \alpha} v^{\prime \beta} v^{\gamma}-\epsilon_{\alpha \beta \gamma \delta} \epsilon_{B}^{\alpha} \epsilon_{D}^{* \beta} v^{\prime \gamma} v^{\delta} v^{\mu}\right] .
\end{aligned}
$$

The combinations of these form factors appearing as helicity amplitudes may be denoted

$$
\begin{aligned}
V_{++}= & \frac{f_{9}}{\sqrt{M m}}, \\
V_{+0}= & \frac{f_{8}}{\sqrt{M m}}, \\
V_{0+}= & \frac{1}{\sqrt{2 M m}}\left(M f_{4}+m f_{5}\right) \\
V_{00}= & \frac{1}{4(M m)^{\frac{5}{2}}}\left\{M m\left(t-M^{2}-m^{2}\right)\left(M f_{4}+m f_{5}\right)-2 k^{2} t\left(m f_{6}+M f_{7}\right)\right. \\
& \left.+M m\left[m\left(t+M^{2}-m^{2}\right) f_{8}+M\left(t-M^{2}+m^{2}\right) f_{9}\right]\right\} \\
S_{00}= & \frac{1}{(2 M m)^{\frac{5}{2}}}\left\{M m\left(t-M^{2}-m^{2}\right)\left[M\left(t-M^{2}+m^{2}\right) f_{4}-m\left(t+M^{2}-m^{2}\right) f_{5}\right]\right. \\
& \left.+2 k^{2} t\left[m\left(t+M^{2}-m^{2}\right) f_{6}-M\left(t-M^{2}+m^{2}\right) f_{7}\right]-4 k^{2} t M m\left(m f_{8}-M f_{9}\right)\right\} \\
S_{0+}= & \frac{1}{\sqrt{4 M m}}\left[M\left(t-M^{2}+m^{2}\right) f_{4}-m\left(t+M^{2}-m^{2}\right) f_{5}\right], \\
A_{++}= & \frac{1}{2(M m)^{\frac{3}{2}}}\left\{M m\left[\left(t-M^{2}-m^{2}\right) f_{10}-2 M m f_{11}\right]-2 k^{2} t f_{12}\right\} \\
A_{0+}= & \frac{1}{2(M m)^{\frac{3}{2}}}\left\{M^{2} m\left(t-M^{2}+m^{2}\right) f_{10}-M m^{2}\left(t+M^{2}-m^{2}\right) f_{11}\right. \\
& \left.+2 k^{2} t\left(M f_{12}-m f_{13}\right)\right\} \\
A_{+0}= & \frac{1}{2(M m)^{\frac{3}{2}}}\left\{M m\left[2 M m f_{10}-\left(t-M^{2}-m^{2}\right) f_{11}\right]+2 k^{2} t f_{13}\right\}, \\
P_{0+}= & \frac{1}{2(M m)^{\frac{3}{2}}}\left\{2 M m\left(M f_{10}+m f_{11}\right)+\left[\left(t-M^{2}+m^{2}\right) f_{12}+m\left(t+M^{2}-m^{2}\right) f_{13}\right]\right\}
\end{aligned}
$$


The labels $V, S, A, P$ reflect the spin-parity (vector, scalar, axial vector, or pseudoscalar) of the virtual $W$, while the subscripts denote the helicities of the $W^{*}$ and $D^{*}$ in the decay of the $\bar{B}^{*}$.

Only amplitudes of a fixed spin-parity enter each dispersion relation. For example, the four $V$ helicity amplitudes enter the vector current dispersion relation for $\Pi^{T}$. Above all the appropriate pair-production thresholds, the contribution to $\operatorname{Im} \Pi_{V}^{T}$ from $\bar{B} \bar{D}, \bar{B}^{*} \bar{D}, \bar{B} \bar{D}^{*}$, $\bar{B}^{*} \bar{D}^{*}$, and $\Lambda_{b} \bar{\Lambda}_{c}$ states is

$$
\begin{aligned}
\operatorname{Im} \Pi_{V}^{T} & \geq \frac{k^{3}}{6 \pi \sqrt{t}}\left[2\left(\left|f_{+}\right|^{2}+\left|V_{0+}\right|^{2}+\left|V_{00}\right|^{2}\right)+t\left(|g|^{2}+|\hat{g}|^{2}+\left|V_{++}\right|^{2}+\left|V_{+0}\right|^{2}\right)\right] \\
& +\frac{k\left(t-t_{-}\right)}{12 \pi t^{3 / 2}}\left[2 t\left|F_{1}\right|^{2}+\left|H_{V}\right|^{2}\right]
\end{aligned}
$$

where we have included an isospin factor $n_{I}=2$ for the mesons, and $k$ is defined in Eq. (2.1). Using Eq. (4.13), it is straightforward to compute the weighting functions $\phi$ for all the $\bar{B}^{*} \bar{D}^{*}$ form factors. For each such $F_{i}$, one obtains a parameterization of the form Eq. (3.8), with the unknown expansion coefficients denoted by $b_{i n}$,

$$
F_{i}(z)=\frac{1}{P_{i}(z) \phi_{i}(z)} \sum_{n=0}^{\infty} b_{i n} z^{n} .
$$

The $\phi$ parameters [see (4.14) or (4.23)] $a, b, c, K$ and the relevant $\chi$ for $\bar{B}^{*} \rightarrow D^{*}$ are given in Table 4. 


\begin{tabular}{|c|c|c|c|c|c|}
\hline$F_{i}$ & $K$ & $\chi$ & $a$ & $b$ & $c$ \\
\hline$V_{++}$ & 96 & $\chi^{T}(+u)$ & 3 & 3 & 1 \\
$V_{+0}$ & 96 & $\chi^{T}(+u)$ & 3 & 3 & 1 \\
$V_{0+}$ & 48 & $\chi^{T}(+u)$ & 3 & 3 & 2 \\
$V_{00}$ & 48 & $\chi^{T}(+u)$ & 3 & 3 & 2 \\
$S_{00}$ & 8 & $\chi^{L}(+u)$ & 1 & 1 & 1 \\
$S_{0+}$ & 8 & $\chi^{L}(+u)$ & 1 & 1 & 1 \\
$A_{++}$ & 24 & $\chi^{T}(-u)$ & 1 & 1 & 1 \\
$A_{0+}$ & 24 & $\chi^{T}(-u)$ & 1 & 1 & 2 \\
$A_{+0}$ & 24 & $\chi^{T}(-u)$ & 1 & 1 & 1 \\
$P_{0+}$ & 32 & $\chi^{L}(-u)$ & 3 & 3 & 1 \\
\hline
\end{tabular}

Table 4. Factors entering Eq. (4.14) or (4.23) for the meson form factors $F_{i}$ in $\bar{B}^{*} \rightarrow D^{*}$.

Substituting this expansion into the dispersion relation (4.21) gives

$$
\sum_{i=0}^{H} \sum_{n=0}^{\infty} b_{i n}^{2} \leq 1 .
$$

Included in the sum are all helicity amplitudes $i=0, \cdots, H$ for processes with the right quantum numbers to couple to the current $J$. It is clear that the constraint on a particular helicity amplitude $F_{i}$ can be strengthened if it is possible to relate the various $b_{i n}$, i.e., if one can relate the form factors. This is accomplished with the help of heavy quark symmetry.

\section{B. Form factors in the Heavy Quark Limit}

In general, form factors are not related by heavy quark symmetry throughout the pair production region [32], but may be related in the semileptonic region. This fact has been exploited to improve constraints on the $B \rightarrow B$ elastic form factor [17 and the dominant $\bar{B} \rightarrow \pi \ell \bar{\nu}$ form factor [11]. The situation in the present case is conceptually analogous, although algebraically more cumbersome. 
In the semileptonic region, the 20 form factors of $\bar{B}^{(*)} \rightarrow D^{(*)}$ reduce to only one in the heavy quark limit, the universal Isgur-Wise function $\xi(w)$, with $\xi(1)=1$. Likewise, the six baryon form factors in $\Lambda_{b} \rightarrow \Lambda_{c}$ reduce to another universal function [33], which we may denote $\zeta(w)$, with $\zeta(1)=1$. Recalling that $r=m / M$, the relation of the helicity amplitudes to $\xi$ and $\zeta$ in the heavy quark limit are given by

$$
\begin{aligned}
f_{+} & =\frac{1}{2} \mathcal{F}_{2}=-\frac{1}{2} \hat{\mathcal{F}}_{2}=-\frac{1}{\sqrt{2}} V_{0+}=V_{00}=\frac{1}{2} P_{0+}=\frac{(1+r)}{2 \sqrt{r}} \xi \\
f_{0} & =\mathcal{F}_{1}=\hat{\mathcal{F}}_{1}=-\sqrt{2} S_{00}=S_{0+}=-A_{0+}=M^{2} \sqrt{r}(1-r)(1+w) \xi \\
g & =-\hat{g}=V_{++}=V_{+0}=\frac{1}{M \sqrt{r}} \xi \\
f & =-\hat{f}=-A_{++}=A_{+0}=M \sqrt{r}(1+w) \xi
\end{aligned}
$$

for mesons, and by

$$
\begin{aligned}
F_{0} & =H_{A}=M(1-r) \zeta \\
F_{1} & =G_{1}=\zeta \\
H_{V} & =G_{0}=M(1+r) \zeta
\end{aligned}
$$

for baryons. The only helicity amplitudes in this list protected by Luke's theorem [3],34] from $1 / M$ corrections at $w=1$ are $f, \hat{f}$, and $G_{1}$.

In the strict heavy quark limit, our dispersion relation constraints become useless because the form factors in Eqs. (5.9) and (5.10) develop an essential singularity due to an infinite number of poles just below threshold [35. The description Eq. (3.8) then contains no information, because the Blaschke factor $P(t)$ goes to zero in the semileptonic region.

For finite masses our parameterizations are well-behaved, and heavy quark relations are valid up to $1 / m$ corrections. In this case, some of the form factors in Eqs. (5.9) or (5.10) will have more constraining parameterizations than others because their Blaschke factors, which reflect the number and positions of sub-threshold resonances, will be larger. If one views the universality of the Isgur-Wise function as arising from the dominance of the essential singularity in each of the form factors, one might expect $1 / m$ corrections to be larger for form factors with fewer sub-threshold poles. Of course, it is always possible that the residues of 
the various poles could conspire to keep $1 / m$ corrections small, since Blaschke factors alone permit [36] any residues consistent with the dispersion relation bounds.

We use the heavy quark relations (5.9) and (5.10) to tighten our bounds on parameterization coefficients. When we use these bounds to quote smaller errors on our parameterizations, we allow for substantial deviations due to $1 / m$ effects, thereby minimizing errors induced by assuming full heavy quark symmetry. However, when we use these bounds to test heavy quark symmetry by constraining the slope or curvature of the Isgur-Wise function, one should bear in mind the possibility that heavy quark violations could be larger in form factors with very few sub-threshold poles (such as $f_{0}, S_{00}$, or $S_{0+}$ ) than in those that are typically measured experimentally.

\section{Bounding Parameterization Coefficients}

We now use the heavy quark relations of the higher resonance helicity amplitudes to improve the constraints. For concreteness, consider the form factor $g(w)$. Near zero recoil, $w=1$, heavy quark symmetry relates it to six other form factors appearing in the dispersion relation,

$$
\begin{aligned}
g(w) & =-\hat{g}(w)=V_{++}=V_{+0} \\
& =\frac{2}{M+m} f_{+}(w)=\frac{2}{M+m} V_{00}=-\frac{\sqrt{2}}{M+m} V_{0+} .
\end{aligned}
$$

While exact heavy quark spin symmetry implies that the functional dependence of these form factors is the same for all values of $w$ corresponding to semileptonic decay, for physical masses we demand only that the normalization and first and second derivatives of these form factors are roughly equal at $w=1$. This suffices to provide lower bounds, assuming full heavy quark symmetry, on the contributions of the form factors in Eq. (5.11) to the sum in Eq. (5.8). Once computed, it is straightforward to weaken these lower bounds by including factors indicating the violation of heavy quark symmetry.

Since each form factor $F_{i}$ in (5.11) has a parameterization of the form (5.7), the coefficients $a_{n}$ in the expansion of $g$ can be related to the coefficients $b_{i n}$ in the expansion of $F_{i}$ 
by

$$
\sum_{n} a_{n} z^{n} \approx \frac{1}{C_{i}(z)} \sum_{n} b_{i n} z^{n},
$$

where $\approx$ means only the normalization and first two derivatives are equal at $w=1$. The functions

$$
C_{i}=\frac{\Xi_{g} P_{i} \phi_{i}}{\Xi_{i} P_{g} \phi_{g}}
$$

are given by ratios of Blaschke factors, weighting functions, and symmetry factors $\Xi_{i}(z)$ chosen so that

$$
\xi(z)=\Xi_{i}(z) F_{i}(z)
$$

in the heavy quark limit. The kinematic factors $\Xi_{i}(w)$ appear in Eqs. (5.9) and (5.10). By choosing the same value of $N$ for each form factor, we ensure that the kinematic variable $z(w ; N)$ is process-independent, most of the $z$ dependence in $\phi_{g}(z) / \phi_{i}(z)$ cancels out, and the $C_{i}(z)$ become quite simple.

Numerically, the values of $N$ that optimize our constraints correspond to $N=1+2 \epsilon$ with $\epsilon \approx 0.05$, and ignoring terms of order $\epsilon^{2}$ in Eq. (5.12) is a good approximation for parameter values $a_{n}$ that saturate their bounds. Evaluating (5.12) and its first and second derivatives at $w=1(z \approx-\epsilon / 2)$, we find for each $i$,

$$
\begin{aligned}
b_{0} & =C a_{0} \\
b_{1} & =C^{\prime} a_{0}+C a_{1} \\
b_{2} & =\frac{1}{2} C^{\prime \prime} a_{0}+C^{\prime} a_{1}+C a_{2}+\frac{3}{2} \epsilon\left(b_{3}-b_{3}^{\mathrm{HQS}}\right),
\end{aligned}
$$

where $C, C^{\prime}=d C / d z$ and $C^{\prime \prime}=d^{2} C / d z^{2}$ are evaluated at $z=0($ not $w=1)$, and $b_{3}^{\mathrm{HQS}}$ is the value $b_{3}$ would have if the third derivative of (5.12) yielded a valid relation. Departures from the heavy quark symmetry limit tend to increase as we take higher derivatives of (5.12), so one might expect substantial corrections to $b_{3}=b_{3}^{\mathrm{HQS}}$. However, corrections to this relation are multiplied by $\epsilon$, so we may justifiably ignore the factor $3 \epsilon\left(b_{3}-b_{3}^{\mathrm{HQS}}\right) / 2$ in $b_{2}$. 
Substituting Eq. (5.15) into

$$
\sum_{n=0}^{\infty} a_{n}^{2}+\sum_{i=1}^{H} \sum_{n=0}^{\infty} b_{i n}^{2} \leq 1
$$

gives more stringent bounds on the coefficients $a_{n}$. For the form factor $g$, they yield $-0.37 \leq$ $a_{1} \leq 0.40$ and $-0.49 \leq a_{2} \leq 0.47$, compared to $\left|a_{1}\right|,\left|a_{2}\right| \leq 1$ from Eq. (3.9). Bounds for the other form factor coefficients can be obtained in a straightforward fashion by singling out a different set of $b_{i n}$ in (5.12).

The bounds on $a_{1}$ are useful as tests of heavy quark symmetry. For example, parameterizing $f_{0}$ and constraining $-0.62 \leq a_{1} \leq 0.58$ restricts the slope of the Isgur-Wise function, up to $1 / m_{c}$ effects, to $-0.3 \leq \rho^{2} \equiv-\left.(d \xi / d w)\right|_{w=1} \leq 1.8$. Bounds on $a_{2}$ are useful for decreasing truncation errors of our one-coefficient parameterizations, as described in the next section. In this case, however, we do not want to rely on exact heavy quark symmetry, because we wish to be as conservative as possible with how much the symmetry improves the truncation error, so we allow for explicit violations to the infinite mass limit. Such violations are potentially largest for the bounds on $a_{2}$, because they depend on relations involving second derivatives. Allowing for the total contribution from the higher-spin states to be as low as $60 \%$ of their infinite-mass value gives bounds on $a_{2}$ of $-0.58 \leq a_{2} \leq 0.57$ for $g$, which leads to a corresponding decrease in the error induced by truncating the expansion (3.8) by $40 \%$ [see Eq. 6.4) below]. In summary, bounds on $a_{1}$ are obtained using full, unbroken heavy quark symmetry, and may be used to test the accuracy of this symmetry. The bounds on $a_{1}$ do not enter the construction of our parameterizations. The derivation of the bounds on $a_{2}$ allows for substantial violations of heavy quark symmetry. These bounds enter into the truncation errors we quote on our parameterizations.

Since the baryonic and mesonic form factors are not related by heavy quark symmetry, Eq. (5.16) only applies in the baryonic case to the pairs $F_{1}, H_{V}$ and $G_{1}, H_{A}$. Of these, only $H_{V}$ and $H_{A}$ receive substantial improvements to their truncation errors, which are proportional to $a_{3}$ [see Eq. (6.4)]. While $1 / m_{c}$ corrections to relations involving $a_{3}$ and $b_{3}$ could à priori be large, they are known in terms of one constant $\bar{\Lambda}_{\Lambda}$ for the baryons [37], 
and are not particularly large. For example, the relation between $H_{V}$ and $F_{1}$ is independent of $w$, to $O\left(1 / m_{c}^{2}\right)$. Allowing for the contributions to the dispersion relation from $F_{1}$ and $G_{1}$ to be as small as $50 \%$ of their heavy quark symmetry values gives $\left|a_{3}\right|_{\max }=0.57$ for $H_{V}$ and $\left|a_{3}\right|_{\max }=0.29$ for $H_{A}$.

The bounds on $a_{1}$ ignoring heavy quark violation, and the bounds on $a_{2}$ allowing for deviations from the heavy quark limit as described above, are given in Tables 5 and 6 .

\section{TRUNCATION ERRORS}

To fit data with our parameterizations, we must truncate the series (3.8) after a finite number $Q$ of unknown coefficients $a_{n}$. This introduces a truncation error which can be minimized by choosing an appropriate value of $N$ [0] (or equivalently, $t_{0}$ ). Rather than use $a_{0}$ as a free parameter as in [8], we solve for $a_{0}$ so that the form factor is automatically normalized at $w=1$ to $F(1)$. If $N=1$, then $z(1 ; N)=0$, and this parameterization coincides with that in [8]. For $N \geq 1$, the parameterization (3.8), including the solution for $a_{0}$, may be re-expressed as

$$
\Xi_{F}(w) F(w)=\frac{\Xi_{F}(w)}{P(w) \phi(w ; N)}\left\{P(1) \phi(1 ; N) F(1)+\sum_{n=1}^{Q} a_{n}\left[z^{n}(w ; N)-z^{n}(1 ; N)\right]\right\},
$$

where the product $\Xi_{F} F$ is normalized to coincide with the Isgur-Wise function $\xi(w)$ [or $\zeta(w)$ for baryons] in the heavy quark limit. The full form factor is of course given by the $Q \rightarrow \infty$ sum, while approximations $F^{\text {fit }}$ are obtained by truncating at finite $Q$; then the fit coefficients $a_{1}, \ldots, a_{Q}$ can be chosen so that the difference between the parameterization $F^{\text {fit }}$ and the actual form factor $F$ is given by

$$
\begin{aligned}
\Xi_{F}(w)\left(F(w)-F^{\mathrm{fit}}(w)\right) & =\frac{\Xi_{F}(w)}{P(w) \phi(w)} \sum_{n=Q+1}^{\infty} a_{n}\left(z^{n}-z_{\min }^{n}\right) \\
& \approx \frac{\Xi_{F}(w)}{P(w) \phi(w)} a_{Q+1}\left(z^{Q+1}-z_{\min }^{Q+1}\right)
\end{aligned}
$$

where we have ignored numerically unimportant higher order terms. Using the Schwarz inequality, the boundedness condition $\sum_{n} a_{n}^{2} \leq 1$, and the geometric series sum, these higher order terms can be shown to be smaller than 


$$
\begin{aligned}
\left|\frac{\Xi_{F}(w)}{P(w) \phi(w)} \sum_{n=Q+2}^{\infty} a_{n}\left(z^{n}-z_{\min }^{n}\right)\right| & \leq\left|\frac{\Xi_{F}(w)}{P(w) \phi(w)}\right| \sqrt{\sum_{n=Q+2}^{\infty}\left|a_{n}\right|^{2} \sum_{n=Q+2}^{\infty}\left(z^{n}-z_{\min }^{n}\right)^{2}} \\
& \leq\left|\frac{\Xi_{F}(w)}{P(w) \phi(w)}\left(z^{Q+2}-z_{\text {min }}^{Q+2}\right)\right|+O\left(z^{Q+4}\right),
\end{aligned}
$$

which, since $|z| \leq 0.04$ for the cases at hand, can be justifiably ignored. Thus, to good accuracy, the maximal possible truncation error $T\left(\Xi_{F} F\right)$ for any physically allowed $z$ is

$$
T\left(\Xi_{F} F\right)=\max \left|\frac{\Xi_{F}(w)\left(z^{Q+1}-z_{\min }^{Q+1}\right)}{P(w) \phi(w)}\right| \cdot\left|a_{Q+1}\right|_{\max },
$$

where $Q$ is the highest power of $z$ used in the parameterization fit and $\left|a_{Q+1}\right|_{\max }$ is the maximal allowed value of $\left|a_{Q+1}\right|$. The inclusion of higher states leads to tighter bounds on $\left|a_{Q+1}\right|_{\max }$ and thus smaller truncation errors.

The truncation error vanishes at $w=1$, where the normalization is fixed. For optimal values of $N, z_{\min } \approx-z_{\max }$, so for one-coefficient parameterizations the truncation error drops sharply for some $w$ near $w_{\max }$. This leads to significantly smaller truncation errors than in previous works [7,8]. For two-coefficient parameterizations, the factor $\left(z^{3}-z_{\min }^{3}\right)$ adds constructively near $w_{\max }$; for a given bound on $\left|a_{3}\right|$, this leads to a larger truncation error than in previous work [7]. This is unavoidable as long as only $a_{1}$ and $a_{2}$ are fit parameters, i.e., as long as $a_{0}$ is chosen to enforce the normalization at zero recoil, so that the truncation error vanishes at $z=z_{\min }$ rather than $z=0$.

Our truncation errors for one- and two-coefficient parameterizations of the various form factors in $\bar{B} \rightarrow D \ell \bar{\nu}, \quad \bar{B} \rightarrow D^{*} \ell \bar{\nu}$, and $\Lambda_{b} \rightarrow \Lambda_{c} \ell \bar{\nu}$ are shown in Tables 5 and 6 . The value of the free parameter $N$ has been optimized for each form factor and number of fit parameters to produce the smallest truncation errors. The bounds on $a_{1}$ come from Eq. (5.16) and the heavy quark symmetry relation Eq. (5.15), and may be used as tests of heavy quark symmetry. The bounds on $a_{2}$ enter our truncation errors, so allowance for heavy quark symmetry violation has been made as described in the previous section. The truncation error is expressed as a percentage of $\Xi_{F}(1) F(1)$, which equals unity in the heavy quark limit. The truncation errors in Table 5 are typically less than half those of previous parameterizations, while the errors in Table 6 are either better or worse by nearly a factor 
of two, depending on the form factor [7] (we have corrected an oversight in this reference, which used incorrect Blaschke factors for $F_{0}$ and $\left.G_{0}\right)$.

Note the especially small size of the truncation error for the form factors $f_{0}$ and $\mathcal{F}_{2}$; if we consider all form factors related by heavy quark symmetry, then the champion in this respect is $S_{0+}$, with a truncation error of only $0.56 \%$. However, as we discuss in the next section, actually fitting data to such form factors introduces much larger $1 / m$ uncertainties.

\begin{tabular}{|c|c|c|c|c|}
\hline$F$ & $N_{\text {optimal }}$ & \multicolumn{2}{|c|}{ Combined bounds from $\bar{B}^{(*)} \bar{D}^{(*)}$} & $T\left(\Xi_{F} F\right)$ \\
\hline$f_{+}$ & 1.108 & $-0.23 \leq a_{1} \leq 0.20$ & $-0.55 \leq a_{2} \leq 0.58$ & $2.6 \%$ \\
$f_{0}$ & 1.109 & $-0.62 \leq a_{1} \leq 0.58$ & $-0.78 \leq a_{2} \leq 0.85$ & $0.7 \%$ \\
\hline$f$ & 1.093 & $-0.37 \leq a_{1} \leq 0.39$ & $-0.58 \leq a_{2} \leq 0.56$ & $1.8 \%$ \\
$\mathcal{F}_{1}$ & 1.093 & $-0.06 \leq a_{1} \leq 0.06$ & $-0.11 \leq a_{2} \leq 0.10$ & $2.1 \%$ \\
$g$ & 1.093 & $-0.37 \leq a_{1} \leq 0.40$ & $-0.58 \leq a_{2} \leq 0.57$ & $1.2 \%$ \\
$\mathcal{F}_{2}$ & 1.093 & $-0.41 \leq a_{1} \leq 0.45$ & $-0.59 \leq a_{2} \leq 0.57$ & $0.6 \%$ \\
\hline$F_{0}$ & 1.081 & $-1 \leq a_{1} \leq 1$ & $-1 \leq a_{2} \leq 1$ & $6.3 \%$ \\
$G_{0}$ & 1.080 & $-1 \leq a_{1} \leq 1$ & $-1 \leq a_{2} \leq 1$ & $9.4 \%$ \\
\hline
\end{tabular}

Table 5. One-coefficient parameterizations using optimized $N$. Bounds on $a_{1}$ ignore heavy quark violation. Bounds on $a_{2}$ allow for violation as described in Sec. $\square$. The truncation error is relative to the normalization of $\Xi_{F} F$ at $w=1$.

For baryon form factors, the large number of sub-threshold poles typically ensures that at least two parameters are required. Even with two parameters and the spin-symmetry improvements, the truncation errors are significant. Using three parameters reduces the truncation errors to negligible levels. 


\begin{tabular}{|c|c|c|c|c|}
\hline$F$ & $N_{\text {optimal }}$ & \multicolumn{2}{|c|}{ Combined bounds from $\bar{B}^{(*)} \bar{D}^{(*)}$} & $T\left(\Xi_{F} F\right)$ \\
\hline$F_{1}$ & 1.104 & $-0.90 \leq a_{1} \leq 0.90$ & $-0.97 \leq a_{2} \leq 0.97$ & $7.0 \%$ \\
$H_{V}$ & 1.104 & $-0.31 \leq a_{1} \leq 0.31$ & $-0.53 \leq a_{2} \leq 0.53$ & $12 \%$ \\
$G_{1}$ & 1.104 & $-0.98 \leq a_{1} \leq 0.98$ & $-0.99 \leq a_{2} \leq 0.99$ & $9.1 \%$ \\
$H_{A}$ & 1.104 & $-0.15 \leq a_{1} \leq 0.15$ & $-0.26 \leq a_{2} \leq 0.26$ & $18 \%$ \\
\hline$F_{0}$ & 1.104 & $-1 \leq a_{1} \leq 1$ & $-1 \leq a_{2} \leq 1$ & $0.35 \%$ \\
$G_{0}$ & 1.104 & $-1 \leq a_{1} \leq 1$ & $-1 \leq a_{2} \leq 1$ & $0.53 \%$ \\
\hline
\end{tabular}

Table 6. Two-coefficient parameterizations using optimized $N$. Bounds on $a_{1}$ ignore heavy quark violation. Bounds on $a_{2}$ allow for violation as described in Sec. $\square$. The truncation error is relative to the normalization of $\Xi_{F} F$ at $w=1$.

A way to circumvent the relatively large truncation errors on most of the baryon form factors is revealed by an interesting feature of Table 5: The $\Lambda_{b} \rightarrow \Lambda_{c} \ell \bar{\nu}$ form factor $F_{0}$ is welldescribed (to about $6 \%$ ) by a one-coefficient parameterization. The contribution from $F_{0}$ to the decay rate is suppressed by the lepton mass, so it is difficult to observe. However, $F_{0}$ is related by heavy quark symmetry to all the other baryon form factors [see (5.10)]. Unlike in meson decays, the relation including $1 / m_{c}$ effects is known in terms of one constant [37] $\bar{\Lambda}_{\Lambda} \approx M_{\Lambda_{b}}-m_{b}$. Thus, the differential decay rate can be described in terms of two constants, $a_{1}$ and $\bar{\Lambda}_{\Lambda}$. Using the value of $\left|V_{c b}\right|$ obtained from $\bar{B} \rightarrow D^{(*)} \ell \bar{\nu}$ and known hadron masses should then allow a determination of $\bar{\Lambda} \approx M_{B}-m_{b}$. This quantity is important because it enters both exclusive and inclusive semileptonic decay distributions as a $1 / m_{c}$ correction.

In addition to errors incurred by truncating the expansion (6.1), there are a number of uncertainties arising from various approximations we have made. We enumerated in [8] a list of uncertainties which must be estimated for a reliable determination of the quality of the dispersive bounds, and found that we could allow for their effects by increasing truncation errors by $40 \%$. Since then, we have greatly reduced or eliminated many of these uncertainties. In [7] we saw that the inclusion of the additional parameter $N$ (or $t_{0}$ ) permits a dramatic reduction of the truncation error, and the more realistic definition of 
the truncation error used here reduces the uncertainties of one-parameter fits even more. The simultaneous inclusion of all $\bar{B}^{(*)} \bar{D}^{(*)}$ states on the hadronic side of the dispersion relation serves to help saturate the bound from the partonic side, and the explicit inclusion of two-loop perturbative and leading nonperturbative effects eliminates them as a source of uncertainty. The uncertainty in $B_{c}$ pole positions is most significant for poles near threshold, and leads to larger truncation errors only if the pole masses have been overestimated. As pointed out in [7], branch cuts from multi-particle states below threshold can be ignored if they violate isospin. The only uncertainty from [8] that has not been reduced is due to the choice of pole quark masses in the perturbative calculation. Combining the remaining uncertainties as in [\&], we find that their effects may be allowed for by increasing $B \rightarrow D, D^{*}$ truncation errors by $20 \%$ (e.g., the conservative truncation error for $f$ would be $2.2 \%$ ) and $\Lambda_{b} \rightarrow \Lambda_{c}$ truncation errors by $30 \%$. In nearly all cases, this small increase makes no practical difference.

\section{SLOPE AND CURVATURE RELATIONS}

A set of interesting relations between the slopes and curvatures of $\bar{B} \rightarrow D \ell \bar{\nu}$ and $\bar{B} \rightarrow D^{*} \ell \bar{\nu}$ form factors has been derived by Caprini and Neubert [16]. Here we examine these relations in the context of the parameterization formalism, point out and circumvent an invalidating assumption, and discuss the utility of the new, valid, relations.

We have seen that each of the form factors $F$ for $\bar{B}^{(*)} \rightarrow D^{(*)} \ell \bar{\nu}$ can be expanded in a series

$$
F(z)=\frac{1}{P(z) \phi(z)} \sum_{n=0}^{\infty} a_{n} z^{n}
$$

where, in order to compare with [16], we have set $t_{0}=t_{-}(N=1)$. From Eq. (3.9), the coefficients $a_{n}$ obey

$$
a_{0}^{2}+a_{1}^{2}+a_{2}^{2} \leq 1
$$

Expressing $a_{0}, a_{1}$, and $a_{2}$ in terms of $z$-derivatives of $P(z) \phi(z) F(z)$ at $z=0$, Eq. (7.2) gives 


$$
\left.[P(z) \phi(z) F(z)]^{2}\right|_{z=0}+\left.\left[\frac{d}{d z}(P(z) \phi(z) F(z))\right]^{2}\right|_{z=0}+\left.\frac{1}{4}\left[\frac{d^{2}}{d z^{2}}(P(z) \phi(z) F(z))\right]^{2}\right|_{z=0} \leq 1
$$

For $t_{0}=t_{-}, z=0$ corresponds to $w=1$. Then Eq. (7.3) constrains the slope $-\rho^{2}$ and curvature $c$ defined by

$$
F(w)=F(1)-\rho^{2}(w-1)+c(w-1)^{2}+O\left[(w-1)^{3}\right]
$$

to lie within an ellipse.

Equation (7.3) is the starting point of reference [16], with one critical simplification: They consider the form factor $\tilde{f}_{0} \equiv f_{0} /\left[\left(M_{B}-M_{D}\right) \sqrt{M_{B} M_{D}}(1+w)\right]$, and argue that it does not receive contributions from scalar $B_{c}$ mesons that would generate poles in $f_{0}$. This allows them to set $P(z)=1$, leading to a nearly linear relation between $\rho^{2}$ and $c$,

$$
c \approx 0.72 \rho^{2}-0.09
$$

Their argument is based on the assumption that the scalar $B_{c}$ mesons are broad resonances because they can decay into two-particle intermediate states such as $B_{c}\left(0^{-}\right)+\eta$. Unfortunately, the very potential models they cite refute this idea. For example, Ref. [29] has the mass of the scalar $2{ }^{3} P_{0}$ state, $6.700 \mathrm{GeV}$, below the two-particle threshold for an $\eta$ plus the $1{ }^{1} S_{0} B_{c}$ ground state, $6.264+0.548=6.812 \mathrm{GeV}$. The scalar $2{ }^{3} P_{0}$ is thus essentially stable with respect to hadronic transitions, since transitions involving one pion are suppressed by isospin, two pions by parity (or phase space, in decays to a $B_{c}^{*}$ ), and three pions by phase space. All the references [28] that calculate the relevant scalar masses and widths agree that there are two narrow, scalar $B_{c}$ resonances below the $\bar{B} \bar{D}$ threshold. It is also worth nothing that the lowest-lying charmonium scalar state $\chi_{c 0}$ is narrow.

While sub-threshold branch cuts from states containing at least a $b$ and a $c$ quark may be legitimately ignored [7,8], it is well known that poles play an essential role in the shape of the form factor [11,35]. For this reason, the slope-convexity relations derived in [16] are invalid. 
New relations can be derived by simply including the two scalar $B_{c}$ states in $P(t)$. We use masses from [29], which agree with other potential model determinations to better than $1 \%$. It is algebraically straightforward to input physical masses and expand our parameterization in powers of $(w-1)$,

$$
\begin{aligned}
\tilde{f}_{0}(w) & =\tilde{f}_{0}(1) \\
& +\left(+1.72 a_{1}-0.77 \tilde{f}_{0}(1)\right)(w-1) \\
& +\left(-1.74 a_{1}+0.21 a_{2}+0.55 \tilde{f}_{0}(1)\right)(w-1)^{2} \\
& +\left(+1.41 a_{1}-0.27 a_{2}-0.38 \tilde{f}_{0}(1)\right)(w-1)^{3} \\
& +\left(-1.03 a_{1}+0.25 a_{2}+0.25 \tilde{f}_{0}(1)\right)(w-1)^{4}+\ldots,
\end{aligned}
$$

and solve for the coefficient $c$ of $(w-1)^{2}$ in terms of the coefficient $-\rho^{2}$ of $(w-1)$. We find

$$
c=1.02 \rho^{2}+0.21 a_{2}-0.23 \tilde{f}_{0}(1) .
$$

This is not a very interesting relation because the unknown coefficient $a_{2}$, which can be as large as $\pm 1(\approx \pm 0.6$ if we include the contribution of higher states and ignore heavy quark symmetry violation), significantly affects the slope-convexity relationship. Had we ignored the Blaschke factor $P(t)$, the coefficient of $a_{2}$ would have been 0.07 .

For $N=1$, our usual truncation analysis shows that $a_{2}$ can contribute at most $4 \%$ to $\tilde{f}_{0}(w)$. This may seem surprising, given its obvious importance in (7.6) and (7.7). The $4 \%$ value arises from a cancellation of the $a_{2}$ dependence among the various $(w-1)^{n}$ coefficients; note the alternating signs of the $a_{n}$ coefficients. The cancellation is not accidental, but reflects the naturalness of an expansion in $z(w ; N)$ rather than $(w-1)$. This effect is highlighted by the observation that the $(w-1)^{3}$ term in (7.6) can be as large as $40 \%$ at $w_{\max }=1.6$, indicating that $\tilde{f}_{0}$ must be expanded to rather high order in $(w-1)$ if percentlevel accuracy is desired.

The expansion (7.6) can, alternatively, be used to test heavy quark symmetry by placing a restriction on the slope $-\rho^{2}$. If we include the contribution from the spin-related states $\bar{B}^{(*)} \bar{D}^{(*)}, a_{1}$ is restricted to $-0.61 \leq a_{1} \leq 0.59$, leading to 


$$
-0.26 \leq \rho^{2} \leq 1.84
$$

The same relation can be derived using the form factors $S_{0+}$ or $S_{00}$. These bounds are somewhat weaker than those derived from Bjorken [4] and Voloshin [5] inequalitiest, which restrict $0.22 \leq \rho^{2} \leq 1.15$ once $O\left(\alpha_{s}\right)$ corrections have been included 38 .

\section{EXPERIMENTAL FITS}

While constraints obtained from unobserved form factors like $f_{0}$ may serve as tests of heavy quark symmetry, they are not well-suited for fitting to data. The reason is that once the truncation error on a form factor is sufficiently small (a few percent), heavy quark symmetry violating effects of order $20-30 \%$ become the main concern. For $\bar{B} \rightarrow D \ell \bar{\nu}$, using the parameterization of $f_{+}$avoids any dependence on heavy quark symmetry. For $\bar{B} \rightarrow D^{*} \ell \bar{\nu}$, using the form factor $f$ minimizes the dependence on heavy quark symmetry. This is because on the one hand, the ratio

$$
\frac{f}{M_{B} \sqrt{M_{B} M_{D^{*}}}(1+w) g}=\frac{\hat{C}_{1}^{5}}{\hat{C}_{1}}\left[1+\frac{\bar{\Lambda}}{2 m_{c}}(w-2)\right]+O\left(\frac{1}{m_{b}}, \frac{1}{m_{c}^{2}}\right) .
$$

is given in terms of a single [3], roughly determined constant $\bar{\Lambda} \approx 300-600 \mathrm{MeV}$ and known [39] perturbative functions $C_{1}^{5} / C_{1}=1+O\left(\alpha_{s}\right)$, while on the other hand the ratio $a_{+} / g=$ $-1 / 2$ is determined using only spin symmetry, which is expected to hold more precisely than full flavor-spin symmetry [40]. We use $f$ rather than $g$ because it is protected from $1 / m$ corrections [河] at zero recoil.

The purpose of the following fits is not to extract the best value of $\left|V_{c b}\right|$, since only the experimental groups themselves can correctly account for efficiencies, resolutions, smearing

\footnotetext{
${ }^{4}$ The physics leading to these results is quite different: The Bjorken and Voloshin inequalities use perturbative QCD to bound exclusive form factors directly in the semileptonic region, while the dispersion constraints use both perturbative QCD in the pair-production region and the phenomenological mass spectrum in the unphysical region $t_{-}<t<t_{+}$.
} 
effects, etc. Rather, since we expect the approximate results from using the QCD-derived parameterization to survive these experimental corrections, these fits may be used to motivate a more thorough analysis.

Form factors for $\Lambda_{b} \rightarrow \Lambda_{c} \ell \bar{\nu}$ may be extracted in the near future at CDF [41] or LEP [42]. Current data for $\bar{B} \rightarrow D^{(*)} \ell \bar{\nu}$ decay spectra are available from CLEO [43], ALEPH [44], OPAL [45], and DELPHI [46]; older data exists from ARGUS [47]. Some progress has been made towards measuring individual form factors in $\bar{B} \rightarrow D^{*} \ell \bar{\nu}$ 48]. When this is finally accomplished, the parameterizations for individual form factors can be applied without recourse to heavy quark symmetry, except for the uncertainties in the value of $F(1)$.

In the meantime, one must rely on the heavy quark symmetry prediction that the $\bar{B} \rightarrow D^{*} \ell \bar{\nu}$ differential rate is proportional to a function $\mathcal{F}(w)$ that is normalized to $1+O\left(1 / m^{2}\right)$ at zero recoil and is proportional to the Isgur-Wise function $\xi(x)=\Xi_{f}(w) f(w)$ up to $1 / m_{c}$ corrections. We may then use the one-coefficient, QCD-derived parameterization of $f$ obtained from (5.9) and (6.1) to extract from data the values of $\left|V_{c b}\right| \mathcal{F}(1)$ and $a_{1} / \mathcal{F}(1)$,

$$
\frac{\mathcal{F}(w)}{\mathcal{F}(1)}=\frac{1}{(1+w) P_{f}(w) \phi_{f}(w ; N)}\left\{2 P_{f}(1) \phi_{f}(1 ; N)+\frac{a_{1}}{\mathcal{F}(1)} \frac{[z(w ; N)-z(1 ; N)]}{\sqrt{M_{B} M_{D^{*}}}}\right\},
$$

where $N=1.093, z(w ; N)$ is defined in Eq. (4.17), $\phi_{f}(w ; N)$ is given by Eq. (4.23) and Table 1, and $P_{f}(w)$ is determined from Eqs. (4.25), (4.16), and the first four vector masses in Table 3.

The procedure is precisely as detailed in [8], except that we now have only one fit coefficient instead of two [7]. Morever, the improvements described above reduce our oneparameter truncation errors to no more than $3 \%$. We fit to the experiments whose differential distributions are easily available. A $\chi^{2}$ per degree of freedom (d.o.f.) fit using our QCD dispersion relation bounds (QCD fit) to CLEO data 43 gives

$$
\begin{aligned}
\text { QCD Fit } \quad 10^{3} \cdot\left|V_{c b}\right| \mathcal{F}(1) & =36.9_{-2.1}^{+2.0}, \\
\frac{a_{1}}{\mathcal{F}(1)} & =0.000_{-0.019}^{+0.022}, \\
\text { Linear Fit } \quad 10^{3} \cdot\left|V_{c b}\right| \mathcal{F}(1) & =35.1_{-1.9}^{+1.9},
\end{aligned}
$$


with $\chi_{\min }^{2}$ /d.o.f. $=0.67$, while a fit to ALEPH data 44 gives

$$
\begin{aligned}
\text { QCD Fit } \quad 10^{3} \cdot\left|V_{c b}\right| \mathcal{F}(1) & =31.9_{-2.4}^{+2.4}, \\
\frac{a_{1}}{\mathcal{F}(1)} & =0.096_{-0.034}^{+0.040}, \\
\text { Linear Fit } \quad 10^{3} \cdot\left|V_{c b}\right| \mathcal{F}(1) & =31.9_{-1.8}^{+1.8},
\end{aligned}
$$

with $\chi_{\min }^{2} /$ d.o.f. $=0.74$. We have also included the values quoted by the experimental groups using a linear fit for comparison, and only statistical errors at one standard deviation are listed.

For $\bar{B} \rightarrow D \ell \bar{\nu}$, we can fit the parameterization of $f_{+}$,

$$
\frac{f_{+}(w)}{f_{+}(1)}=\frac{1}{P_{f_{+}}(w) \phi_{f_{+}}(w ; N)}\left\{P_{f_{+}}(1) \phi_{f_{+}}(1 ; N)+\frac{a_{1}}{f_{+}(1)}[z(w ; N)-z(1 ; N)]\right\},
$$

directly to data, without the need to invoke heavy quark symmetry. Here $N=1.108$ and $P_{f_{+}}$depends on the first three vector masses in Table 3.

In this case, a fit to ALEPH data [4] yields

$$
\begin{aligned}
\text { QCD Fit } \quad 10^{3} \cdot\left|V_{c b}\right| \mathcal{F}_{D}(1) & =29.2_{-7.3}^{+7.3,} \\
\frac{a_{1}}{\mathcal{F}_{D}(1)} & =0.095_{-0.066}^{+0.110}, \\
\text { Linear Fit } \quad 10^{3} \cdot\left|V_{c b}\right| \mathcal{F}_{D}(1) & =27.8_{-6.8}^{+6.8},
\end{aligned}
$$

with $\chi_{\text {min }}^{2}$ d.o.f. $=1.94$, and now $\mathcal{F}_{D}(1)=\Xi_{f_{+}}(1) f_{+}(1)=2 \sqrt{r} f_{+}(1) /(1+r)$.

For CLEO $\bar{B} \rightarrow D^{*} \ell \bar{\nu}$ data, which exhibits discernible curvature, our central values of $\left|V_{c b}\right| \mathcal{F}(1)$ lie at the upper $1 \sigma$ boundary of the linear-fit result, while for ALEPH data, which is extremely flat, the central values are nearly the same. The statistical errors are larger because the QCD parameterization allows for curvature.

The ALEPH $\quad \bar{B} \rightarrow D \ell \bar{\nu}$ data presents an interesting area in which to test our results: The shape of their data will have to change as the statistical errors are reduced, if it is to be consistent with QCD.

\footnotetext{
${ }^{5}$ The larger $\chi^{2} /$ d.o.f. is due to their binned data point at $w=1.55$, which suggests a peculiar upturn of the form factor near $w_{\max }$.
} 


\section{CONCLUSIONS}

Form factors can be reliably bounded in the pair-production region of momentum-space by perturbative QCD calculations. Analyticity, crossing symmetry, and dispersion relations may then be used to translate these bounds into constraints in the phenomenologically interesting semileptonic region.

While these constraints typically imply rather weak bounds on the slopes of form factors 10, 17, 18, 36, quite stringent bounds can be obtained if the form factor at two or more points is known. The constraints actually imply an infinite number of increasingly stringent bounds, depending on the number of points at which the form factor is known [10,12, All of these bounds are automatically obeyed if the form factor is parameterized as in Eq. (3.8), even if the parameterization is truncated after a few terms.

In this paper, we have eliminated some of the uncertainty involved in the derivation of these parameterizations by including two-loop perturbative corrections to the partonic side of the dispersion relation. We have also presented parameterizations for form factors whose contribution to semileptonic decay rates is suppressed by the lepton mass.

We examined a relation between the slope and curvature of the Isgur-Wise function derived by Caprini and Neubert [16], and pointed out the presence of sub-threshold singularities that invalidate their analysis. Once these singularities are correctly accounted for,

the slope-convexity relations become rather weak. Bounds on the slope of the Isgur-Wise function made by ignoring finite mass corrections are also fairly weak. We point out that even with strong constraints on the slope and curvature, higher-order terms in a $(w-1)$ expansion of the Isgur-Wise function can be quite large. The parameterization in Eq. (3.8) does not suffer from this limitation.

For $b \rightarrow c$ transitions, we reduced the truncation errors that describe the accuracy of such parameterizations. This was accomplished in part by using a parameterization that is automatically normalized at zero recoil to a quantity $\mathcal{F}(1)$, which must be supplied by some other method such as heavy quark symmetry. More importantly, we included the 
contributions of higher states in the hadronic side of the dispersion relation. These states, the $\bar{B}^{*} \bar{D}$ and $\bar{B}^{*} \bar{D}^{*}$ pairs, are related to $\bar{B} \rightarrow D \ell \bar{\nu}$ and $\bar{B} \rightarrow D^{*} \ell \bar{\nu}$ form factors in the semileptonic region by heavy quark symmetry. Even in the presence of substantial heavy quark symmetry violation, these relations place lower bounds on the contribution from the higher states to the dispersion relation that lead to tighter upper bounds on the magnitudes of the unknown parameterization coefficients. This in turn reduces the truncation errors on the parameterizations of the various form factors.

For most of the $\bar{B} \rightarrow D, D^{*}$ form factors, the inclusion of higher states reduces truncation errors by roughly $40 \%$. In one case, $\mathcal{F}_{1}$, the truncation error is reduced by as much as a factor of ten. This hefty improvement arises because $\mathcal{F}_{1}$ contributes very little to the dispersion relation, so it is far from saturating the perturbative bound until its spin-symmetry partners are included as well. After including all $\bar{B}^{(*)} \bar{D}^{(*)}$ states, we find that each of the six form factors governing $\bar{B} \rightarrow D \ell \bar{\nu}$ and $\bar{B} \rightarrow D^{*} \ell \bar{\nu}$ is described to better than $3 \%$ accuracy using only one unknown parameter. This should be a considerable aid in experimentally disentangling the various form factors in differential decay distributions.

For $\Lambda_{b} \rightarrow \Lambda_{c} \ell \bar{\nu}$ decays, there are no spin-symmetry partners to help saturate the dispersion relation bound. However, the presence of more than one helicity amplitude in the same bound achieves the same effect. This is most dramatic in the case of $H_{A}$, whose truncation error is reduced by a factor of four. Of greater interest is the observation that the form factor $F_{0}$ can be described at the $6 \%$ level using only one unknown coefficient. Because the $1 / m_{c}$ corrections to heavy quark symmetry relations among the baryonic form factors are given in terms of one additional parameter, $\bar{\Lambda}_{\Lambda}$, the entire decay distribution can be described using only two unknown constants. This should allow a relatively clean extraction of the phenomenologically interesting quantity $\bar{\Lambda}_{\Lambda}$.

Finally, we used a parameterization of the form factor $f$ and heavy quark relations to extract $\left|V_{c b}\right|$ from $\bar{B} \rightarrow D^{*} \ell \bar{\nu}$ data. This choice of form factor minimizes the dependence on heavy quark symmetry. We expect the qualitative features of this extraction to persist even after the effects of experimental resolution, smearing, etc., are properly incorporated. Our 
analysis suggests that the implicit error associated with the choice of parameterization is comparable to the statistical errors normally quoted. Similar statements apply to $\bar{B} \rightarrow D \ell \bar{\nu}$, except that in this case no reliance on heavy quark symmetry is necessary, since we can parameterize the form factor $f_{+}$directly.

Further improvements may be possible by including additional higher states in the dispersion bound, or perhaps by weighing the dispersion integral differently. One could also readily incorporate approximate $S U(3)$ symmetry by using an effective $n_{I}=2.5$ isospin factor [16 in $\bar{B} \rightarrow D, D^{*}$ decays, which would decrease truncation errors by an additional 10\%. While such improvements would be welcome for the baryonic form factors, their util-

ity for $\bar{B} \rightarrow D, D^{*}$ form factors is not clear. This is because, once truncation errors are at the few-percent level, the overwhelming source of uncertainly comes from heavy quark symmetry violations, which are expected to be of order $30 \%$. Such uncertainties highlight the importance of extracting individual form factors, which can be parameterized using one coefficient without recourse to heavy quark symmetry.

\section{Note Added}

The essential role of the scalar $B_{c}$ poles in the slope-convexity relations of Caprini and Neubert has been pointed out independently by L. Lellouch (private communication). A corrected slope-convexity relation and other topics related to those in this work are in preparation by these authors [49]. Additional criticism of the neglect of the scalar poles appears in Refs. [50].

\section{Acknowledgments}

We would like to thank André Hoang, Bernd Kniehl, Karl Schilcher, and Sotos Generalis for discussions concerning higher-order QCD results, Aneesh Manohar for discussions about the partial wave decomposition, and Martin Savage and Lawrence Gibbons for comments on statistical errors. This work is supported by the Department of Energy under contracts 
Nos. DOE-FG03-97ER40506 and DOE/ER/40682-132. 


\section{REFERENCES}

[1] N. Isgur and M. B. Wise, Phys. Lett. B 232, 113 (1989); B 237, 527 (1990);

M. B. Voloshin and M. A. Shifman, Yad. Fiz. 47, 801 (1988) [Sov. J. Nucl. Phys. 47, $511(1988)]$.

[2] E. Eichten and B. Hill, Phys. Lett. B 234, 511 (1990).

[3] M. E. Luke, Phys. Lett. B 252, 447 (1990).

[4] J. D. Bjorken, in Proceedings of the 4th Recontres de Physique de la Vallèe d'Aoste, La Thuille, Italy, 1990, ed. M. Greco (Editions Frontières, Gif-Sur-Yvette, 1990);

N. Isgur and M. B. Wise, Phys. Rev. D 43, 819 (1991).

[5] M.B. Voloshin, Phys. Rev. D 46, 3062 (1992).

[6] I.I. Bigi, M.A. Shifman, N.G. Uraltsev, and A.I. Vainshtein, Phys. Rev. D 52, 196 (1995);

C. G. Boyd and I. Rothstein, Phys. Lett. B 395, 96 (1997).

[7] C. G. Boyd and R. F. Lebed, Nucl. Phys. B 485, 275 (1997).

[8] C. G. Boyd, B. Grinstein, and R. F. Lebed, Nucl. Phys. B 461, 493 (1996).

[9] C. G. Boyd, B. Grinstein, and R. F. Lebed, Phys. Lett. B 353, 306 (1995).

[10] C. G. Boyd, B. Grinstein, and R. F. Lebed, Phys. Rev. Lett. 74, 4603 (1995).

[11] C. G. Boyd and M. J. Savage, Report No. CMU-HEP97-02, DOE/ER/40682-127, DOE/ER/41014-07-N97 hep-ph/9702300 (unpublished).

[12] L. Lellouch, Nucl. Phys. B 479, 353 (1996).

[13] D. Becirevic, Report No. LPTHE-Orsay 96/14 hep-ph/9603298 (unpublished).

[14] D. Becirevic, Phys. Rev. D 54, 6842 (1996).

[15] I. Caprini, Z. Phys. C 61, 651 (1994); Phys. Lett. B 339, 187 (1994). 
[16] I. Caprini and M. Neubert, Phys. Lett. B 380, 376 (1996).

[17] I. Caprini and C. Macesanu, Phys. Rev. D 54, 5686 (1996).

[18] I. Caprini, Phys. Rev. D 52, 6349 (1995); Phys. Rev. D 534082 (1996).

[19] C. Bourrely, B. Machet, and E. de Rafael, Nucl. Phys. B 189, 157 (1981).

[20] S. C. Generalis, J. Phys. G 16, 785 (1990).

[21] L. J. Reinders, S. Yazaki, and H. R. Rubinstein, Phys. Lett. B 97, 257 (1980); 103, 63 (1981); Phys. Rep. 127, 1 (1985).

[22] A. Djouadi and P. Gambino, Phys. Rev. D 49, 3499 (1994).

[23] For a description of the problem and references, see M. Jamin and M. Münz, Z. Phys. C 60, 569 (1993).

[24] S. C. Generalis, J. Phys. G 16, 367 (1990).

[25] E. Witten, Nucl. Phys. B 104, 445 (1976); B 122, 109 (1977);

D. J. Broadhurst and S. C. Generalis, Phys. Lett. B 139, 85 (1984).

[26] S. Narison, QCD Spectral Sum Rules in Lecture Notes in Physics, Vol. 26, World Scientific, Singapore, 1989.

[27] R. M. Barnett et al. (Particle Data Group), Phys. Rev. D 54, 1 (1996).

[28] S. S. Gershtein, V. V. Kisilev, A. K. Likhoded, and A. V. Tkabladze, Phys. Rev. D 51, $3613(1995)$;

Y.-Q. Chen and Y.-P. Kuang, Phys. Rev. D 46, 1165 (1992).

[29] E. J. Eichten and C. Quigg, Phys. Rev. D 49, 5845 (1994); C. Quigg, private communication.

[30] E.J. Eichten, C. T. Hill and C. Quigg, Phys. Rev. Lett. 71, 4116 (1993); FERMILABCONF-94/117-T, published in the Proceedings of the CHARM2000 Workshop, Fermi- 
lab, June 1994; FERMILAB-CONF-94/118-T (unpublished).

[31] C. G. Boyd and B. Grinstein, Nucl. Phys. B 451, 177 (1995). The $\bar{B}^{*} \rightarrow D^{*}$ form factors are defined only in the electronic version, hep-ph/9502311.

[32] P. Ball, H. G. Dosch, and M. A. Shifman, Phys. Rev. D 47, 4077 (1993).

[33] N. Isgur and M. B. Wise, Nucl. Phys. B 348, 276 (1991).

[34] C. G. Boyd and D. E. Brahm, Phys. Lett. B 257, 392 (1991);

R. F. Lebed and M. Suzuki, Phys. Rev. D 44, 829 (1991).

[35] E. Carlson, J. Milana, N. Isgur, T. Mannel, and W. Roberts, Phys. Lett. B 299, 133 (1993);

A. Falk, M. Luke, and M. B. Wise, Phys. Lett. B 299, 123 (1993);

B. Grinstein and P. Mende, Phys. Lett. B 299, 127 (1993);

C. Dominguez, J. Körner, and D. Pirjol, Phys. Lett. B 301, 373 (1993).

[36] E. de Rafael and J. Taron, Phys. Rev. D 50, 373 (1994).

[37] H. Georgi, B. Grinstein, and M. B. Wise, Phys. Lett. B 252, 456 (1990).

[38] A.G. Grozin and G.P. Korchemsky, Phys. Rev. D53 (1996) 1378;

C. G. Boyd, B. Grinstein, and A. V. Manohar, Phys. Rev. D 54, 2081 (1996);

C. G. Boyd, Z. Ligeti, I. Z. Rothstein, and M. B. Wise, Phys. Rev. D 553027 (1997).

[39] A. Falk and B. Grinstein, Phys. Lett. B 249, 314 (1990);

M. Neubert, Phys. Rep. 245 (1994) 259;

[40] B. Grinstein, in Proceedings of the Workshop on B Physics at Hadron Accelerators, Snowmass, Colorado, 1993, eds. C.S. Mishra and P. McBride (Fermilab, 1994).

[41] CDF Collaboration, http://www-cdf.fnal.gov/physics/new/bottom/cdf3353/cdf3353 .html. 
[42] ALEPH Collaboration, contribution to the International Europhysics Conference on High Energy Physics, Brussels, Belgium, July 1995, EPS0753;

P. Abreu et al. (DELPHI Collaboration), Z. Phys. C 68, 375 (1995);

R. Akers et al. (OPAL Collaboration), Phys. Lett. B 353, 402 (1995).

[43] B. Barish et al. (CLEO Collaboration), Phys. Rev. D 51, 1014 (1995).

[44] D. Buskulic et al. (ALEPH Collaboration), Phys. Lett. B 395, 373 (1997).

[45] K. Ackerstaff et al. (OPAL Collaboration), Phys. Lett. B 395, 128 (1997).

[46] P. Abreu et al. (DELPHI Collaboration), Z. Phys. C 71, 539 (1996).

[47] H. Albrecht et al. (ARGUS collaboration), Z. Phys. C 57, 533 (1993).

[48] A. Anastassov et al., CLEO Collaboration, CLEO CONF 96-8, ICHEP96 PA05-079, in Proceedings of the 28th International Conference on High Energy Physics, Warsaw, 25-31 July 1996 (World Scientific, Singapore, 1997).

[49] I. Caprini, L. Lellouch, and M. Neubert, CPT-97/P.3480, CERN-TH/97-91.

[50] I. Bigi, M. Shifman, and N. Uraltsev, hep-ph/9703290 (unpublished);

N. Uraltsev, Nucl. Instrum. Meth. A384, 17 (1996). 